\title{
e-Phaïstos
}

e-Phaïstos Revue d'histoire des techniques / Journal of the history of technology

VII-1 | 2019

Le travail humain

\section{Le lutage des collections en fluide : histoire d'une technique entre pratique de cabinet et expériences de terrain}

Sealing of Fluid-Preserved Collections: History of a Technique between Practice of Cabinet and Field Experiences

\section{Amandine Péquignot}

\section{OpenEdition}

Journals

Édition électronique

URL : http://journals.openedition.org/ephaistos/4563

DOI : $10.4000 /$ ephaistos. 4563

ISSN : 2552-0741

Éditeur

IHMC - Institut d'histoire moderne et contemporaine (UMR 8066)

Référence électronique

Amandine Péquignot, "Le lutage des collections en fluide : histoire d'une technique entre pratique de cabinet et expériences de terrain », e-Phaïstos [En ligne], VII-1 | 2019, mis en ligne le 15 avril 2019, consulté le 10 décembre 2020. URL : http://journals.openedition.org/ephaistos/4563 ; DOI : https:// doi.org/10.4000/ephaistos.4563

Ce document a été généré automatiquement le 10 décembre 2020.

Tous droits réservés 


\title{
Le lutage des collections en fluide : histoire d'une technique entre pratique de cabinet et expériences de terrain
}

\author{
Sealing of Fluid-Preserved Collections: History of a Technique between Practice \\ of Cabinet and Field Experiences
}

Amandine PEQUIGNOT

\begin{abstract}
Autant les cabinets et les voyages naturalistes sur la période XVII-XVIII siècles en France sont des objets d'études très documentés et analysés en histoire des sciences ${ }^{1}$, autant les techniques de préparation des collections naturalistes le sont dans une moindre mesure. Or, la création du spécimen d'histoire naturelle, depuis la collecte de l'animal à sa mise en collection, offre un angle d'étude particulier unissant l'histoire des techniques aux enjeux scientifiques et muséologiques des collections, et permettant d'appréhender le phénomène dans toute sa globalité. Les techniques de préparation, et en particulier celles liées aux collections d'histoire naturelle en fluide, reflètent une dichotomie entre, d'une part les pratiques des voyageurs-naturalistes confrontées à la réalité du terrain, et d'autre part celles des naturalistes-sédentaires accumulant et étudiant les curiosités ramenées par les premiers. Nous avons choisi d'étudier plus particulièrement celle du lutage des bocaux qui illustre, à son échelle, la confrontation des méthodes théoriques des uns à l'expérimentation des autres, et la contribution de la pratique de terrain aux changements d'un savoir-faire original, permettant au final d'esquisser une première chrono-typologie.
\end{abstract}

\section{L'usage du lut en chimie et distillerie}

2 Le lut, du latin lutum (boue, limon, terre de potier, argile) est un enduit de composition variable qui en séchant, vise soit à boucher hermétiquement des interstices et des 
récipients pour éviter une éventuelle fuite ou évaporation, soit renforcer des objets pour les protéger de l'action directe du feu. Dans la première édition de son Dictionnaire (1694), l'Académie limitait l'usage du lut (boue) aux activités des chimistes, et le définissait comme la «matière dont ils se servent pour boucher les vases qu'ils mettent au feu $»^{2}$. En effet, dès le XVII ${ }^{e}$ siècle, ces derniers l'employaient pour cimenter les jointures de la verrerie utilisée pour leurs expériences (distillation, combustion, dissolution, fermentation) afin d'empêcher l'air de s'y infiltrer et les vapeurs de s'en échapper. Dans l' Encyclopédie de Diderot et D'Alembert, le lut était également lié au domaine de la chimie et définissait :

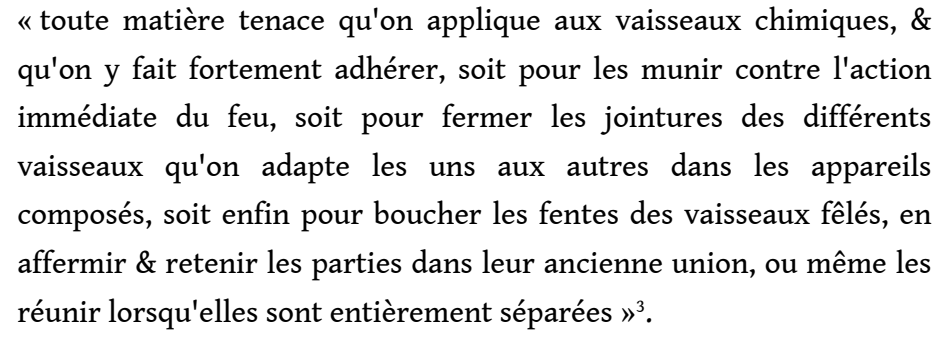

3 La composition du lut dépend de la nature des vapeurs engendrées au cours des réactions chimiques qui peuvent l'altérer. Pour de simples vapeurs aqueuses et non corrosives, le lut simple se limitait à des bandes de papier, de linge ou de vessie de porc mouillées et enduites de colle de farine $e^{5}$ Lorsque les vapeurs se trouvaient plus agressives et dissolvantes, le mélange se composait d'une pâte liquide de chaux et de blanc d'œuf, étalée sur des bandes de linge enroulées aux niveaux des jointures des verreries. Le lut prenait alors le nom de lut sec ou lut de chaux et de blanc d'œuf. Enfin, face à des vapeurs salines, acides ou hautement corrosives, les chimistes avaient recours au lut gras, réalisé à partir d'argile très sèche, réduite en poudre fine et humectée pour donner une pâte à laquelle de l'huile de lin cuite était ajoutée. Après que cette pâte façonnée en cylindres était appliquée aux jointures et séchée, elle était recouverte de bandes de linge enduites de lut sec maintenues avec un lien.

D'autres corps de métiers, comme les distillateurs et liquoristes, eurent recours aux luts pour emboiter et ajuster au mieux les appareils et verreries, et préserver la santé des personnes au contact d'éventuelles vapeurs alcooliques. L'un d'entre eux, Nicolas Lebeaud, réserva un chapitre intitulé «Lutage des appareils » dans son Manuel complet théorique et pratique du distillateur et du liquoriste $e^{6}$, «abrégé commode et que l'on peut consulter avec confiance » selon la Revue Encyclopédique ${ }^{7}$, Lebeaud y lista les principaux luts : ceux empruntés aux chimistes et apothicaires, précédemment cités, comme le lut de papier et de vessie (lut simple), le lut de calcaire et de blanc d'œuf (lut sec) et le lut gras auquel il y remplaça l'huile de lin par du vernis d'ambre jaune. Il recensa d'autres luts dont la diversité vint de la substitution de certains ingrédients au grès des usages. Ainsi, le lut alcalin albumineux élaboré avec du sang de boeuf et des cendres de bois, devint le lut calcaire albumineux en substituant la chaux hydratée aux cendres. Le lut calcaire et au blanc d'œuf, quant à lui, ne différa du précédent qu'en remplaçant le sang par du blanc d'œuf. Furent mentionnés également le lut de cire jaune, le lut de farine de froment brulé utilisée dans les distilleries de pommes de terre et de grain. Lebeaud fit remarquer la bonne tenue de la farine de lin ou de celle de la moutarde avec de la colle d'amidon, et de la résistance à la pression de la pâte d'amendes. Le lut fut aussi mentionné pour d'autres registres d'activités pour coller des faïences ou porcelaines brisées, des auges de pierre ou robinets 
fêlés, ou pour boucher des bouteilles. Pour ce dernier cas, le lut se composa d'un mélange fondu de cire, de saindoux et de térébenthine en proportion égale.

\section{Le lut dans les collections d'histoire naturelle}

5 Fermer hermétiquement un récipient pour limiter les évaporations d'un liquide fut également la préoccupation des naturalistes, en raison des nombreux spécimens d'histoire naturelle plongés dans un liquide conservateur, afin de les étudier. Selon leur taille et leur forme, ceux-ci pouvaient être placés dans une fiole (petite bouteille de verre), une flûte (bouteille en verre de forme étroite et allongée), un bocal (forme cylindrique, pourvu d'un col court et d'un large orifice), un vase (récipient en métal, en porcelaine, en terre, en verre ou en marbre) ou un vaisseau (vase de quelque matière que ce soit, destiné à contenir des liquides). Ces collections -dîtes «en fluide »- détenaient une place importante aux XVII ${ }^{e}$ et XVIII ${ }^{e}$ siècles au sein des cabinets en France ou en Europe ${ }^{8}$. Pour ne citer que quelques exemples parisiens, Joseph Bonnier de la Mosson (1702-1744), riche aristocrate et collectionneur, possédait dans son premier cabinet d'histoire naturelle, le Cabinet des Animaux en phioles [sic] près de 420 bocaux dans lesquels étaient conservés plus de 1000 animaux de toutes sortes (serpents, insectes, oiseaux, quadrupèdes, poissons, monstres et fœetus), et quelques fruits ou plantes étrangères ${ }^{9}$. 


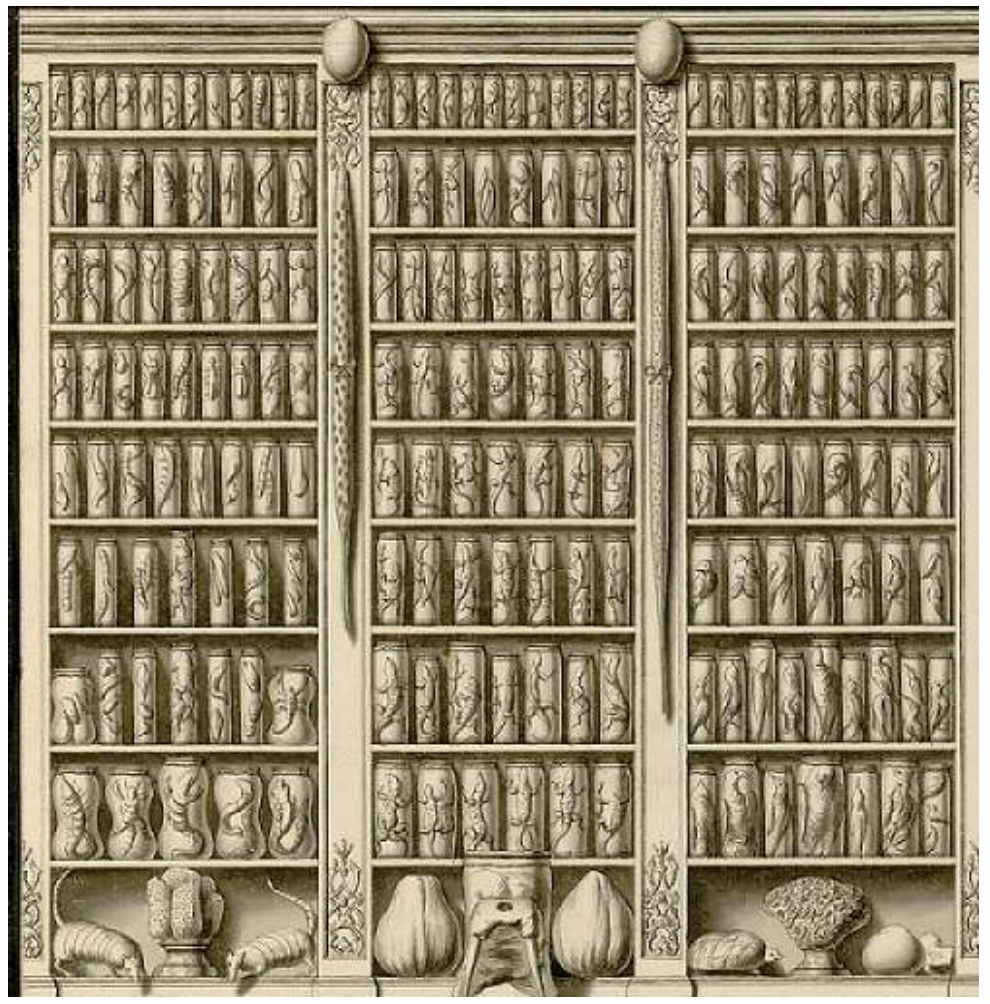

Les bocaux contenant les spécimens sont alignés sur des tablettes dans un ensemble de six grandes armoires. L'organisation semble assez précise et répétitive, ainsi les 6 premières étagères contiennent toujours le même nombre de vases à savoir (de haut en bas) : 12, 8, 9, 9, 8 et 7 . Cette séquence amplifie l'effet de profusion des collections. En revanche, les 2 dernières étagères font apparaître un nombre aléatoire de bocaux de taille et de forme variables, voire insolite (bilobée). Le dessin laisse également deviner l'emploi de vessie comme lutage. Détail de la planche PI.2, 1er cabinet d'histoire naturelle ou cabinet des animaux en fioles, [vue de détail, droite].

(c) Droits réservés - Source Bibliothèque numérique de l'INHA / Collection Jacques Doucet

6 A la même période, on dénombrait dans le Cabinet du Roy du Jardin des Plantes Médicinales : 24 pièces d'anatomie humaine et 210 quadrupèdes (entiers ou en pièces anatomiques) ${ }^{10}$ en esprit de vin. Conserver un spécimen dans un liquide semblait aisé à l'époque, or en pratique et avec le temps, le fluide employé -l'esprit de vin ${ }^{11}$-, et ses vapeurs très volatiles eurent raison des premiers enthousiasmes. Pour combattre cette évaporation naturelle, tout l'enjeu fut d'assurer une herméticité totale et infaillible des récipients :

«[...] tout le monde ne connaît pas le soin et la dépense qu'exige une grande quantité de pièces qui y sont renfermées pour pouvoir les conserver : la plupart des insectes, des poissons, les pièces anatomiques qu'on veut garder dans leur fraicheur et leur souplesse, doivent être tenues dans des vaisseaux de verres remplis d'esprit de vin [...], cette subtile liqueur a toujours trouvé le moyens de s'évaporer malgré les bouchons, les luts et les autres obstacles qu'on a tenté de lui opposer ${ }^{12}{ }^{2}$.

En tant que Garde et Démonstrateur du Cabinet du Roy (1745-1791), le naturaliste bourguignon Louis-Jean-Marie Daubenton (1716-1800), connut cette difficulté et consacra dans l'Histoire naturelle (1749) plus d'une vingtaine de pages sur les moyens de conserver les spécimens en fluide et des luts ${ }^{13}$, reprises en grande partie dans l'Encyclopédie méthodique $(1784)^{14}$. Il y relata ses expériences avortées et fit également le constat que : 
«[...] [1]es luts, les pâtes, les ciments, les mastics que l'on a employez [sic], jusqu'ici, n'ont pas été suffisants pour arrêter la vapeur de l'esprit de vin, soit qu'ils en aient été dissous, soit que cette vapeur humectant continuellement les bords du vase, ait seulement empêché l'adhésion des gommes, de la cire et des graisses que l'on y a appliquées car ayant fait ajuster sur des bocaux des couvercles de verre, j'ai tenté inutilement de fermer le joint avec la cire, il m'a toujours été impossible d'y réussir, il se formait en peu de temps malgré toutes mes précautions un nouveau joint entre la cire et le verre, et je voyais suinter l'esprit de vin $»^{15}$.

Daubenton inventoria et illustra les différentes techniques en sa connaissance (fig. 2) comme tout abord celle d'Henri-Louis Duhamel du Monceau (1700-1782) qui consistait en une plaque de métal apposée sur l'ouverture du bocal, et percée de deux trous par lesquels passèrent deux tuyaux, fermés par des bouchons de liège (fig.1 et 2); le tout mastiqué de céruse délayée dans l'huile grasse des peintres, et recouvert d'une vessie.

Figure 2. Planche V, p.192. DAUBENTON Louis-Jean-Marie, L'Histoire Naturelle générale et particulière : avec la Description du Cabinet du Roy, III, Paris, Imprimerie Royale, 1749

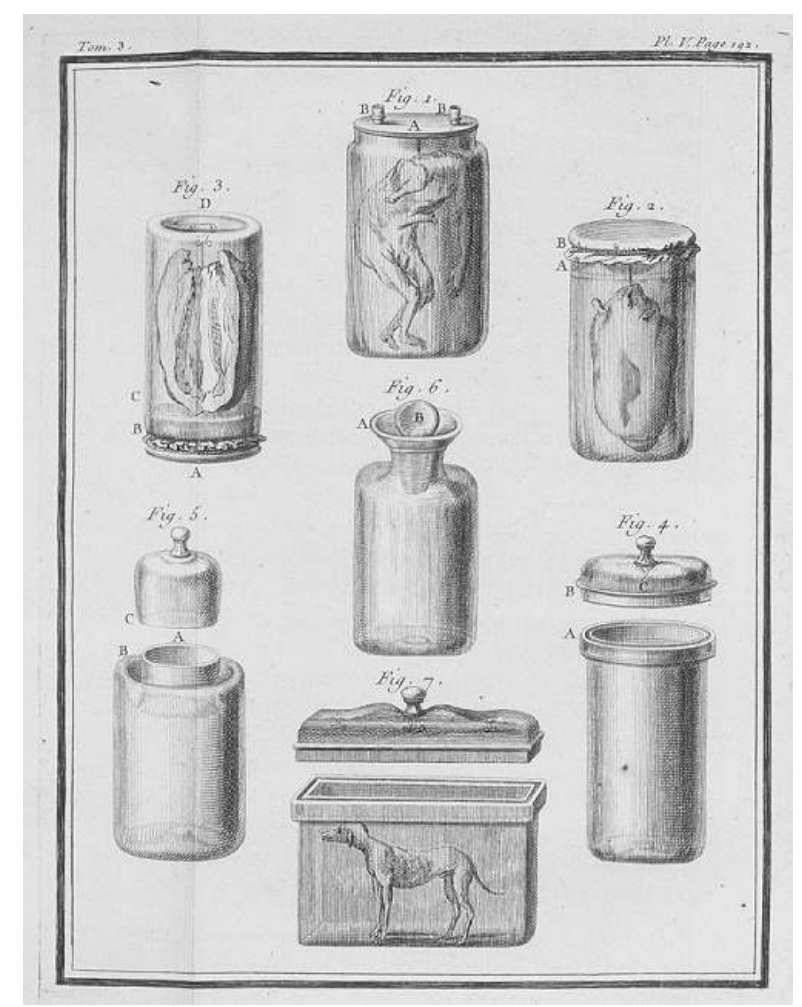

Daubenton a pris soin de faire réaliser une planche sur les différents bocaux et système de lutage en usage pour conserver les collections en fluide. Cette planche tranche avec l'ensemble des planches présentent dans l'Histoire naturelle ; elle est la seule à ne pas décrire des espèces animales ou à représenter des items de la collection du Cabinet du Roy (animaux, squelettes ou parties ostéologiques, organes, etc.).

(c) Droits réservés - Source gallica.bnf.fr / Bibliothèque nationale de France.

9 Toutefois, si sa technique facilitait le renouvellement en alcool des bocaux, Duhamel était conscient que celle-ci ne stoppait pas son évaporation. Daubenton évoqua celle de ClaudeNicolas Le Cat (1700-1768), chirurgien à Rouen et correspondant de l'Académie royale des Sciences. Celui-ci envoya à l'Académie un bocal réalisé par ses soins pour être présenté : les bords de ce bocal étaient creusés en forme de gouttière dans laquelle avait été versé de 
l'huile ou du mercure de sorte qu'étant fermé le bocal ne laissait aucun vide (fig.2 et 4). Cette technique fut employée auparavant par l'alchimiste allemand Johann Rudolph Glauber (1604-1670) qu'il la publia dans son ouvrage traduit en français, les Nouveaux fourneaux philosophiques ou l'Art distillatoire (1659) ${ }^{16}$ (fig.3) et dont Daubenton reproduisît l'illustration (fig.2 et 5).

Figure 3. Planches issues de GLAUBER Johann Rudolph, La description des nouveaux fourneaux philosophiques, ou Art distillatoire, Partie I, Paris, Thomas Jolly, 1659.
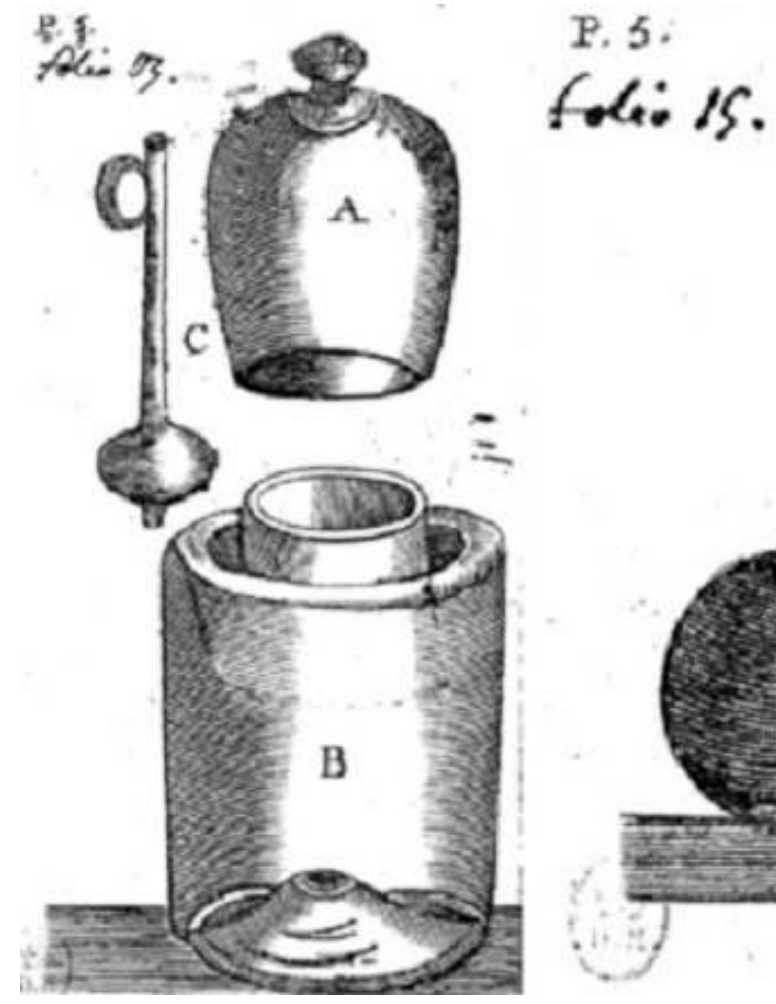

Illustration d'un bocal à bords creusés en forme de gouttière, inventé par le chirurgien rouennais Claude-Nicolas Le Cat, dans laquelle était versée de l'huile ou du mercure de sorte qu'étant fermé le bocal ne laissait aucune possibilité (à gauche) et exemple de flacon anglais dont le bouchon conique en verre vient s'adapter au goulot légèrement évasé de la fiole (à droite).

(c) Droits réservés-Source gallica.bnf.fr / Bibliothèque nationale de France. 
Figure 4. Thesaurus animalium primus, Amstelaedami, Apud Joannem Wolters, 1710 et Opera omnia anatomico-medico-chirurgica, Amstelaedami, Apud Joannem Wolters, 1720

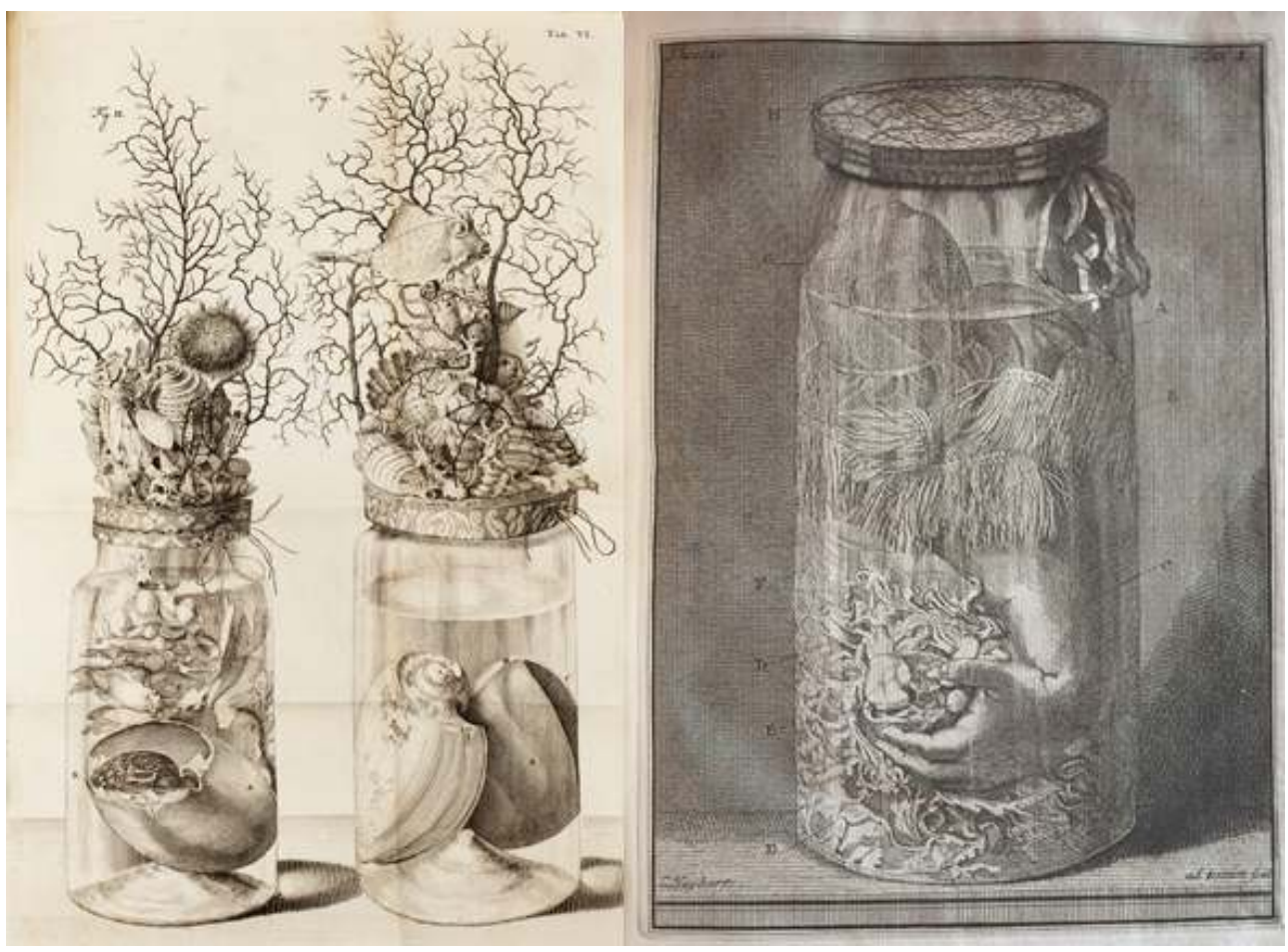

Diversité des lutages de la collection du médecin néerlandais Frederick Ruysch, d’une part ces " vanitas arts » ou Ruyschiana recouverts d'un decorum naturaliste, et d'autre part, des bocaux fermés pour un lutage composé d'une feuille de schiste argileux collée avec de la résine, recouverte d'une vessie de porc tendue, et maintenue par un ruban.

(c) Droits réservés-Source Internet Archives / Smithsonian Libraries et Getty Research Institute. 
Figure 5. SEBA Albertus, Locupletissimi rerum naturalium thesauri accurata descriptio, et iconibus artificiosissimis expressio, per universam physices historiam : Opus, cui, in hoc rerum genere, nullum par exstitit, Vol.1, Amstelaedami : Apud J. Wetstenium, \& Gul. Smith, \& JanssonioWaesbergios, 1734.

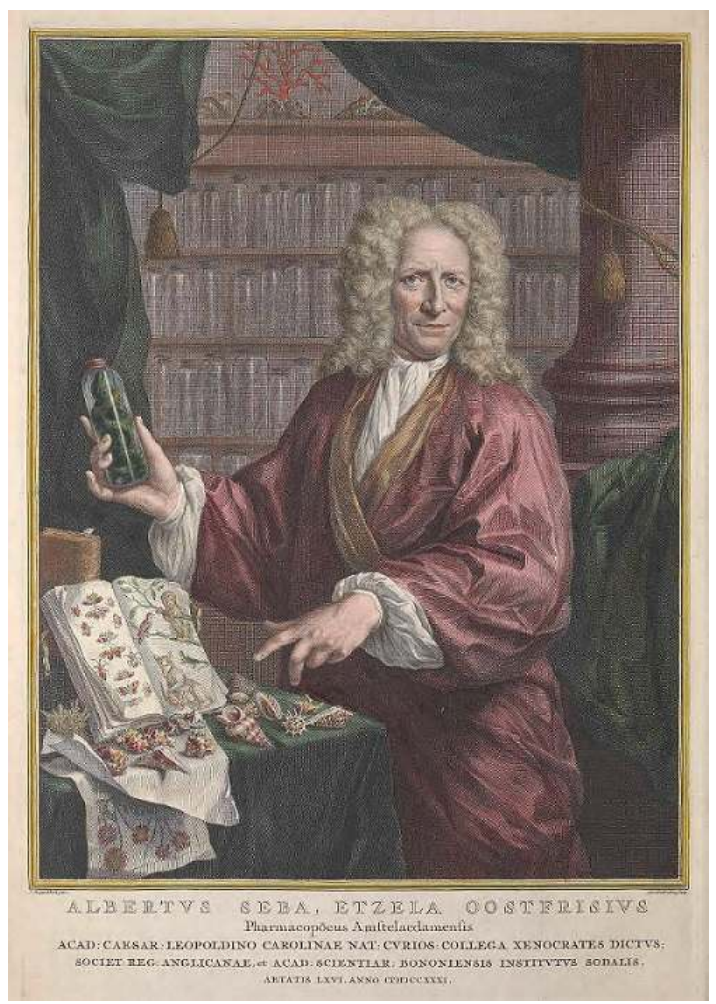

Portrait d'Albertus Seba mis en scène dans son cabinet. Devant une armoire où est stockée plus d'une vingtaine de boucaux, il présente à l'observateur quelques échantillons de sa collection sèche de conchyologie, une planche de son ouvrage (où l'on peut deviner une sarigue) et ses collections en fluide dont notamment un bocal contenant un serpent qu'il tient en main. La mise en couleur des planches de l'ouvrage par Coquebert de Montbret sur les indications de Seba montre un lutage de couleur rouge qui indiquerait l'emploi de cire rouge comme il était d'usage à l'époque.

(c) Droits réservés - Source Internet Archives / Smithsonian Libraries.

Présent également sur cette planche, un exemple de flacon anglais dont le bouchon conique s'adaptait à un goulot légèrement évasé (fig.2 et 6). Le naturaliste sembla suivre les méthodes proposées par le physicien, René-Antoine Ferchault de Réaumur (1683-1757), car «[la] recherche d'une manière de fermer les vaisseaux destinés à conserver des pièces d'histoire naturelle, de façon à interdire tout évaporation à l'esprit de vin, était donc un problème qui méritait toute l'attention des physiciens ; et c'est ce qui a déterminé $\mathrm{M}$. de Réaumur à en tenter la solution ${ }^{17}{ }^{1}$. Les travaux de Réaumur dataient de 1746, mais ne furent publiés qu'en $1751^{18}$ en raison du retard d'impression des Mémoires de l'Académie (1746) comme il tint à le préciser. On comprend sa surprise de lire un extrait détaillé de ses travaux dans l'Histoire naturelle (1749):

«M. Daubenton a fait entrer dans ce volume un discours dans lequel il s'est proposé de traiter à fond des moyens d'empêcher l'évaporation des liqueurs spiritueuses ; c'est-àdire qu'il a placé cet extrait que je ne m'attendais pas d'y trouver, quoique j'eusse informé par M. de Fouchy, qu'il lui avait donnée une copie collationnée de mon Mémoire. Je ne sais si j'en dois des remerciements à $\mathrm{M}$. Daubenton, à qui je ne puis supposer l'intention d'en avoir rien voulu faire qui me fût désagréable : mais il n'est pas douteux que 
l'Académie n'ait lieu d'en être mécontente ; car elle ne saurait manquer de voir quelles pourraient être les conséquences d'un pareil exemple s'il soit imité $»^{19}$.

Dans un premier temps, Réaumur pensait que l'évaporation pouvait être limitée par 1 'amélioration de la forme des vases et des bouchons en cristal. Mais les propositions s'avérant trop dispendieuses, le naturaliste du examiner un autre expédient, celui des propriétés des huiles grasses. En effet, les naturalistes notèrent que les vins d'Italie arrivés en France étaient souvent dans des bouteilles dont le goulot était plein d'huile. Surnageant à la surface, l'huile isolait l'alcool de l'air et rendait impossible la libération des vapeurs alcooliques. Le bouchon du bocal était du liège enduit de suif fondu, et recouvert d'un parchemin (fig. 2). Appliquant ce modus operandi aux collections naturalistes, la réussite ne fut pas de même : la qualité de l'extraction de l'huile fut mise en cause car en réagissant avec l'alcool, elle donnait naissance, selon Réaumur, à de disgracieux et fâcheux " petits flocons de neige » au fond du vase et troublait le liquide. Loin d'être découragé par ce revers, Réaumur aborda le problème sous un angle nouveau et suggéra de remplir le bocal de quelques centimètres d'huile ou de mercure, puis d'y ajouter l'esprit de vin. Le bocal finalement scellé (fig. 2 et 3),

[o]n le pose sur son couvercle, l'huile tombe par ce renversement sur le couvercle qui est devenu le fond du vase et par conséquent l'esprit de vin est au-dessus de l'huile ; dans cette position, [rapporte Réaumur], les vapeurs sont retenues comme dans un vaisseau scellé hermétiquement ${ }^{20}$.

12 L'enjeu fut alors de trouver un moyen d'empêcher l'huile de suinter. Il suffit, selon le naturaliste, d'un parchemin bien ficelé et enduit d'une couche de céruse broyée à la colle, et d'y appliquer plusieurs couches de vernis composé de gomme. Toutefois lorsque le volume du bocal devenait important, le poids du liquide obligeait à mettre un bouchon de liège toujours recouvert d'un parchemin enduit d'un «lut fait avec des matières convenables", mais de composition inconnue. Nombreux furent les naturalistes, concernés par l'accumulation et la conservation des collections en fluide dans les cabinets, se lançant dans des expériences, testant et réfutant des hypothèses, quitte parfois à se perdre en conjecture, et à s'éloigner de la question initiale. Parfois, ils empruntèrent les méthodes des distillateurs qui conservaient leurs liqueurs dans des bouteilles fermées par du liège recouvert d'une couche de blanc de Troyes ${ }^{21}$ délayé dans une pâte de farine, et protégé d'un parchemin. Cette technique fut employée par Daubenton qui sembla lui avoir été bénéfique pour des bocaux de large diamètre. Personnellement, il lui mêlait une teinture d'aloès pour écarter les insectes. Ce lut était ensuite couvert d'un parchemin mouillé, lié fortement autour du bocal. Mais selon Daubenton de toutes les propositions, le mercure fut « le meilleur ». L'usage d'un mélange mercure-plomb ${ }^{22}$, appliqué sur les plaques de verre couvrant l'ouverture des bocaux, fut généralisé par Daubenton à l'ensemble de la collectio:

«L'amalgame de mercure et de plomb est employé pour fermer les bocaux du cabinet d'histoire naturelle du Jardin du roi : si je n'avais pas trouvé ce moyen [...], il n'aurait guère été possible d'avoir un très grand nombre de bocaux $~^{23}$.

13 L'amalgame de Daubenton trouva le soutien de la Commission temporaire des arts, dirigée par le médecin-chirurgien Félix Vicq-d'Azyr (1748-1794), démonstrateur suppléant en anatomie au Jardin du roi et ami de Daubenton, qui souligna dans son Instruction (1794) que « [son] usage est un des nombreux services rendus par ce citoyen aux sciences qu'il cultive $»^{24}$. L'amalgame fut recommandé, mais sa nature non divulguée à l'époque. En revanche, quatre autres méthodes furent régulièrement citées et d'usage par les 
naturalistes ${ }^{25}$ : celle qui consista à fermer les vases d'un parchemin collé et verni avec une dissolution épaisse de cire d'Espagne ${ }^{26}$ dans de l'alcool pur, celle -employée avec plus de succès- du bouchon de liège trempé dans une composition liquide de trois quart de cire et un quart de suif, celle d'un simple disque de liège enduit d'un mélange liquide chaud (quatre parties de brai, une partie de soufre et une demi partie de suif) ou encore celle du rond de verre d'un certain docteur Sue qui consistait en une plaque de verre couverte successivement d'un morceau de parchemin huilé, d'un morceau de plomb laminé, d'un second parchemin trempé dans de l'huile colorée au noir de fumée, et le tout lié autour $\mathrm{du}$ bocal avec une très fine corde.

Certains naturalistes de cabinet, ayant visiblement trouvés le lut parfait, l'exploitèrent, le dissimulèrent ou l'embellirent selon les goûts pour le décorum de leurs antres. Tel fut le cas de la collection du médecin néerlandais Frederick Ruysch (1638-1731) ${ }^{27}$ qui compta plus de 880 bocaux $^{28}$. Dans son Thesaurus anatomicusnonus (1714), Ruysch représenta sur ses planches des pièces anatomiques conservées dans des bocaux dont le lutage serait composé d'une feuille de schiste argileux collée avec de la résine, et recouverte d'une vessie de porc tendue ${ }^{29}$, et dont le pourtour était paré d'un ruban, type gallon à motifs, soie rouge ${ }^{30}$ - . De même, dans son Thesaurus animalium primus (1710), il dévoila des animaux conservés dans des flacons arborant sur leur couvercle ces compositions artistiques et naturalistes, décorum bien connu. Sans nier cette particularité, il ne faudrait limiter la collection Ruysch à cet aspect esthétique souvent qualifié de « vanitas arts » ou de Ruyschiana par certains ${ }^{31}$, puisque $85 \%$ des préparations furent des objets d'étude anatomique sans décoration (Figure 4), essentiellement utilisés à des fins d'études scientifiques $^{32}$. Cette dichotomie entre science et spectacle au sein des collections naturalistes, s'exprimant dans le cas particulier des collections en fluide au niveau de leur lutage, s'observait également sur le frontispice du Thesaurus animalium primus réalisé par Joannem Wolters (fig. 6), où au premier plan, aux pieds des deux dieux Cérès et Saturne ${ }^{33}$, six fioles au lutage fantaisiste tranchaient avec les nombreux bocaux au lutage classique alignés dans le meuble-cabinet au second plan. 


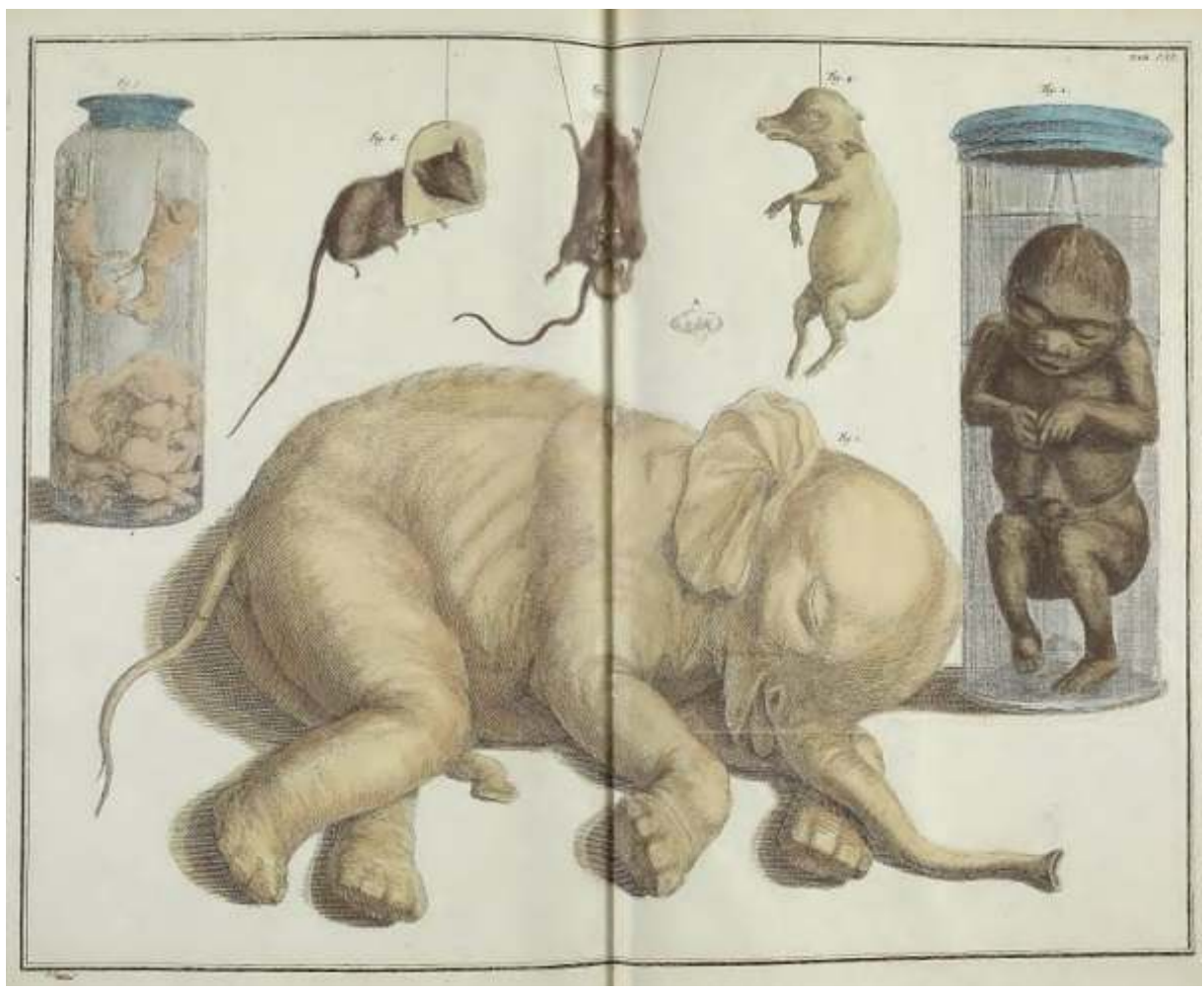

Parmi ces représentations des « spécimens de collections » du cabinet d'Albertus Seba, deux bocaux contenant pour l'un, un fœtus humain (fig.2) et le second, un lot d'embryons (fig.3), la mise en couleur fait apparaître cette fois-ci un lutage de couleur bleutée qui traduit davantage un souci d'esthétisme bien plus qu'une approche réaliste.

(c) Droits réservés - Source Internet Archives / Smithsonian Libraries.

Le zoologiste et pharmacien hollandais Albertus Seba (1665-1736) fut également connu pour son abondante collection d'histoire naturelle. Dans le premier volume de son ouvrage Locupletissimi Rerum Naturalium Thesauri (1734), un portrait ${ }^{34}$ le mettait en scène dans son cabinet tenant dans sa main droite un bocal, devant une accumulation de fioles au lutage simple. Originalement en noir et blanc, la mise en couleur des planches de l'ouvrage (1734-1765) par Antoine-Jean Coquebert de Montbret (1753-1825) sur les indications données par Albertus Seba ${ }^{35}$, fit apparaître des lutages de couleur rouge qui laisserait penser à l'emploi de cire rouge comme il était d'usage à l'époque (fig. 5). Dans l'ouvrage, une planche attire l'attention parmi toutes celles dont la composition artistique et très esthétique illustre le courant des cabinets de curiosités ou « room of wonder ${ }^{36}$ ». La planche Tab.CXI du volume premier (fig. 6) met le lecteur face à la réalité des « spécimens de collections » à travers une représentation crue de différents embryons et fœetus entiers : de porc (fig.4), de rongeurs (fig.6) dont un disséqué (fig.5) accompagné de son embryon extrait (A), d'éléphant posé sur le flanc, et deux bocaux contenant pour l'un, un fœetus humain (fig.2) et le second, un lot d'embryons (fig.3). Dans le cas particulier, la mise en couleur fait apparaitre un lutage composé d'un parchemin curieusement bleuté, maintenu par une ficelle. Il semblerait que pour cette planche, l'esthétisme général de l'ouvrage ait prévalut sur le réalisme, une représentation brute de la pratique quotidienne des collections en fluide. 
$16 \mathrm{Au}$ sein des cabinets d'histoire naturelle, qu'il fut simple fermeture ou support de décorum, le lutage resta une préoccupation pour les collectionneurs qui, faute d'avoir trouvé le plus probant, se trouvèrent souvent impuissants devant l'évaporation des fluides conservateurs et la dégradation progressive des spécimens.

\section{Le Lithocolle de Péron (1810), du voyage au muséum}

Le problème du lutage n'affecta pas seulement les naturalistes sédentaires, mais surtout les naturalistes-voyageurs qui rapportaient avec une certaine difficulté les spécimens collectés, comme le souligna Etienne-François Turgot (1721-1789), naturaliste, administrateur de Malte et gouverneur de Guyane :

«Un grand nombre de Savants ou de simples Curieux travaillent à rassembler les productions naturelles des différents climats ; mais les Correspondants auxquels ils sont obligés de s'adresser dans les Colonies, et ailleurs, [...] ont souvent peine avec la meilleure volonté à bien remplir les commissions qu'on leur donne et les Curieux font quelques fois dans le cas de recevoir à grands frais des choses mal choisies et mal conditionnées, qu'ils sont obligés de mettre au rebut $\|^{37}$.

Dans son Mémoire instructif pour les voyageurs (1758), Turgot aborda cette contrainte sans toutefois apporter des solutions. Seule la figure B de la planche VIII des reptiles présente un bocal contenant un serpent dans une liqueur, dont la légende « On aperçoit comment par-dessus le bouchon on a mis une manière capable d'empêcher la dissipation de la liqueur » resta très succincte. Ce que ne manqua pas de souligner John Coakley Lettsom (1744-1815), médecin et philanthrope anglais, qui jugea les méthodes de Turgot « prolixes et impraticables ${ }^{38} »$. Toutefois celui-ci ne s'aventura pas non plus à partager des méthodes de conservation et emprunta aisément celles de Tesser Samuel Kuckahn (d.1776) publiées en $1770^{39}$. Autant au cours du XVIIIe siècle, les instructions aux voyageurs et les guides furent courants pour lister les objets à collecter, les observations à réaliser ${ }^{40}$, autant les instructions sur les manières de collecter, de préparer, et d'envoyer les spécimens furent peu mentionnées ou détaillées. Il fallait attendre le retour des voyageurs pour voir les recommandations s'étoffer et se préciser.

19 François Péron (1775-1810) et Charles-Alexandre Lesueur (1778-1846) furent de ces naturalistes-voyageurs propulsés dans les grandes épopées, réelles entreprises aléatoires, difficiles, et souvent dangereuses, dont les enjeux les dépassaient parfois et les conditions de collecte leur étaient totalement inconnues. Au mieux, on les conseillait et leur procurait le matériel nécessaire, au pire ce dernier n'était pas du tout adapté et on comptait sur leur débrouillardise. De retour de leur long périple dans les terres australes (1800-1803 $)^{41}$ et forts d'une expérience acquise sur le terrain, Péron et Lesueur décidèrent de publier leur « Mémoire sur la conservation des diverses espèces d'animaux dans l'alcool » dans le Journal de Physique (1810) ${ }^{42}$.

Créée par Jean-Claude de la Métherie (1743-1817), cette revue scientifique était le lieu de présentations de travaux scientifiques, d'échanges, de débats, et parfois de vives invectives entre naturalistes par publications interposées. A travers ce média scientifique, les deux naturalistes souhaitèrent s'adresser directement aux futurs voyageursnaturalistes, qui comme eux, seraient confrontés à la réalité du terrain et aux difficultés de rapporter en France dans de bonnes conditions des animaux. Celles-ci dépendaient de contraintes d'ordre pratique à savoir, la place restreinte dans les navires qui imposait une 
rationalisation des besoins vitaux de l'équipage au dépend du matériel naturaliste de collecte et de conservation, la disponibilité des ressources et du matériel sur place pour suppléer le manque dès le départ, ou enfin la vigilance à apporter aux collections pour survivre aux aléas de telles pérégrinations. Aussi dans cet article, les naturalistes partagèrent le pragmatisme dont ils durent faire preuve pour simplifier des méthodes connues, créer de nouvelles ou suppléer celles de l'industrie pour proposer de nouveaux procédés dont la garantie du succès demeurait les spécimens revenus intactes en France. A travers une anecdote personnelle, Péron ${ }^{43}$ exposa l'attention toute relative accordée au matériel nécessaire à la collecte :

« Lors de ma nomination à l'une des places de zoologiste de l'expédition de découvertes, toute les fournitures nécessaires aux travaux de naturalistes se trouvaient déjà terminées ; et cependant on n'y avait compris ni vases d'aucune espèce, ni alcool, ni rien de ce qui pouvait servir aux collections [...]».

Ses requêtes auprès du chef d'expéditions restèrent lettre morte ${ }^{44}$. Péron doit son salut à Antoine-François de Foucroy (1755-1809), directeur du Muséum ${ }^{45}$, qui l'autorise à acheter le nécessaire en son nom, soit 200 bocaux de verre qui furent embarqués au Hâvre. Par malchance, ceux-ci furent détruits peu de temps après le départ, une des ancres qui avait rompu ses amarres et roulé sur les caisses, en ayant brisé 127.

De ce préjudice, s'entama une réflexion sur les vases à employer pour ne pas ruiner les espoirs d'une expédition naturaliste. Les deux naturalistes testèrent alors les vases de bois qui montrèrent très rapidement des limites. Indépendamment de la difficulté d'en trouver, la première fut que l'alcool se colorait rapidement au contact du bois, dénaturant et noircissant en conséquence les animaux conservés. Ces vases en bois facilitant l'évaporation des alcools, forcèrent une surveillance accrue des caisses et du niveau de fluides, et en conséquence de lourdes manutentions pour les remplir de nouveau. Cette perte de temps et de volume d'alcool -synonyme d'augmentation des dépenses-, obligèrent Péron et Lesueur à abandonner rapidement ce choix et de s'orienter vers les vases de terre. Ces poteries trouvèrent les suffrages des naturalistes, mais leur prix excessif du fait de leur provenance de Chine ou d'Europe, rendit ces recours précaires. Parfois, comme le souligna Péron, il fut impossible de s'en procurer localement, comme par exemple au Timor où ils durent employer des vases non-vernissés réalisés par les habitants pour des usages domestiques. Pour limiter l'absorbance de l'alcool par cette céramique poreuse, les deux naturalistes empruntèrent le brai gras ${ }^{46}$ des marins qui ne donna pas de résultats satisfaisants (l'alcool attaquant la résine).

La solution tint en un savant mélange chauffé de suif et d'huile, et d'en enduire l'intérieur des céramiques. En séchant, les pores étaient ainsi colmatés. Malgré ces essais divers, les vases en verre restèrent les contenants privilégiés avec somme toute de nombreux inconvénients: leur coût d'acquisition élevé d'autant plus important dans les pays lointains, la difficulté d'en réunir une quantité suffisante, leurs formes et tailles souvent non adaptées à la diversité des spécimens collectés, la pauvre qualité du verre, et surtout leur fragilité. Bien entendu, les vases d'apparat -communs dans les cabinets d'histoire naturelle et de curiosités- furent proscrits pour privilégier des verres épais, souvent lourds, mais résistants aux longs transports à terre pour atteindre l'embarcation, aux encombres de longues navigations et aux changements d'arrimage des navires. Un compromis devait être nécessaire entre la solidité et l'économie; les vases de verre noir auraient été de bons candidats si leur opacité permettait d'observer les spécimens. Toujours ayant à l'esprit l'aspect pratique du terrain, les naturalistes recommandèrent de 
limiter la taille des vases à employer : trop grands, leur poids ne faciliterait pas leur transport, requérant en conséquence la mobilisation de plusieurs personnes, et des moyens de locomotion locaux.

Surmonté le problème du contenant, le second point crucial fut la fermeture des vases. Là encore, les deux voyageurs durent distinguer la réalité du terrain et les belles méthodes de cabinet qu'on leur avait exposées avant leur départ. La première d'entre elles fut la " Méthode du Muséum » utilisée dans la galerie d'anatomie de M. Cuvier. D'ordinaire, les vases y étaient fermés par une plaque de verre maintenue avec du mastic de vitrier, ou parfois de la cire grasse rouge ou verte, et l'ensemble revêtu d'une pièce de parchemin mouillé. Péron identifia six inconvénients à cette méthode " rigoureusement impraticable à bord des vaisseaux ${ }^{47}$ ». Il fit l'amère expérience que les disques en verre emportés depuis la France étaient trop fragiles pour résister aux tensions des flacons de grande taille, et le polissage de la surface ne permettait pas à la cire d'y adhérer correctement.

Enfin, les conditions de navigation, comme le simple petit roulis, faisaient sauter aisément ce type de couvercle. Même si le mastic de vitrier adhérait mieux au verre lorsqu'il était bien sec, son séchage était impraticable en pleine mer et obligeait de le faire dans le temps imparti à terre. Dans l'éventualité que le temps des relâches sur terre aurait permis sa bonne tenue, combien allaient se rompre sous la force de l'évaporation pendant les grandes chaleurs comme il était souvent le cas dans les galeries du Muséum, se demanda Péron. Enfin, il souligna le jaunissement intense de ce mastic, susceptible à bord des navires, de se rancir et devenir excessivement friable en raison de son contact répété avec l'alcool dont la température était rarement en dessous de $25^{\circ} \mathrm{C}$. Il fut tout aussi critique sur l'usage du parchemin, qui ne pouvait résister aux conditions particulières qui règnent dans les cales d'un navire (une humidité excessive, de fortes chaleurs et une haute teneur en sel), et moisissait rapidement. Ainsi, tous les moyens de conservation qu'on lui avait prescrits en Europe se trouvèrent en défaut dans les conditions extrêmes du voyage que ce fut le transport des caisses, les mouvements brusques des arrimages, ou le soin tout relatif de l'équipage envers les collections ; « ...il fallait, nous le répétons, trouver un remède direct à ce mal et nous le trouvâmes " ${ }^{48}$.

Tout abord, ils remplacèrent le disque obturateur en verre par un bouchon de liège, moins dispendieux et facile à se procurer. Ce bouchon d'un emploi facile et rapide, sembla être rarement attaqué par l'alcool, et résister aux plus forts roulis et violentes tempêtes. Enfin, pour le sceller durablement, il proposa un nouveau mastic qu'il nomma lithocolle ${ }^{49}$. Le mélange s'obtenait en faisant fondre la cire jaune et la résine, puis l'ocre rouge. Après que l'ensemble eut bouilli pendant un quart d'heure, on versait l'huile de térébenthine, et le tout était porté à ébullition pendant huit à dix minutes. Selon si l'on souhaitait un lut plus ou moins gras, on ajoutait plus ou moins de résine et d'oxyde de fer, ou d'huile de térébenthine et de cire. Pour s'assurer de la qualité du lut préparé, il suffisait de laisser couler sur une assiette ou tout autre corps froid, et on appréciait alors son véritable « degré de force, de ténacité, de mollesse, de fluidité " ${ }^{50}$.

Péron ne révéla pas les quotités des différents composants de son lithocolle, non dans l'espoir de garder sa formule sous le sceau du secret comme il en était de coutume à l'époque, mais afin de laisser les naturalistes libres d'adapter la recette au grès du contexte de travail (musée, laboratoire, voyages-naturalistes), de la disponibilité du matériel, des moyens de chacun et des conditions environnementales. D'une réalisation assez simple, son emploi le fut tout autant: après avoir ajusté le bouchon de liège au diamètre du vase, le mastic encore chaud était appliqué sur le bouchon et le rebord du 
bocal au moyen d'un pinceau. Lorsque le mastic était froid, l'opération était réitérée autant de fois pour obtenir un lut épais, homogène et sans crevasse. Dans le cas particulier des petits bocaux, Péron plongeait directement l'extrémité du vase bouché dans le lithocolle, et l'ôtait rapidement.

Lors de la parution de sa note (1810), Péron prit connaissance du mastic des graveurs grâce à $\mathrm{M}$. Laugier, et eut l'honnêteté de dire que son lithocolle pouvait s'y apparenter. La composition (partie égale de résine et de sable), lui conférait une "dureté prodigieuse ", mais qui avait les inconvénients d'être trop sec et trop fragile, le sable rendait le général grain trop grossier et le mastic ne pouvait alors pénétrer les pores et les fissures du liège, et la résine seule était trop sensible aux actions de l'alcool. Même si Péron semblait avoir découvert le lut miraculeux, il n'en demeurait pas moins prudent, envisageant d'autres moyens pour la prévention des risques :

«Sans doute des bocaux fermés avec tant de précaution offriraient déjà, dans les cas ordinaires, une solidité suffisante pour rassurer le naturaliste le plus vigilant et le plus inquiet; mais toutes les chances les plus défavorables doivent être prévenues à bord des vaisseaux, et notre propre expérience ne nous a que trop appris qu'elles étaient susceptibles de se réaliser toutes $»^{51}$.

Pour couvrir et protéger le lut, il abandonna l'usage du parchemin qui pouvait se putréfier, au profit de la toile qu'il trempa dans de l'huile ou du brai gras. Il porta également une attention particulière à la fermeture des vases -notamment les plus grands- au moyen d'un savant ficelage noué autour du corps et réalisant une croix audessus du bouchon déjà luté et toilé :

"Sans doute les divers moyens que nous venons d'exposer sont minutieux, mais l'habitude en rend la pratique aussi prompt que facile, et les avantages que le zoologiste en retire, le dédommagent bien amplement des soins qu'ils exigent ; en effet les bocaux préparés de cette manière peuvent impunément être renversés sur euxmêmes; ils résisteront à toutes les secousses de la tempête; ils supporteront sans peine les trajets les plus longs; sur de tels vases l'effet de la chaleur sera, sinon tout-à-fait insensible, du moins peu considérable, l'évaporation, le fléau redoutable du collecteur dans les régions équatoriales, ne saurait plus lui causer que de faibles dommages $»^{52}$.

Péron avoua :

«[...] [c]'est en usant de ces diverses précautions et de plusieurs autres qu'il nous reste à faire connaître, que nous sommes parvenues nous-mêmes, au milieu des circonstances les plus désastreuses, à préparer ces belles et nombreuses collections alcooliques dont nous avons enrichi la science et la patrie $»^{53}$.

31 Au sujet de leur voyage dans les terres australes, un rapport signé par Laplace, Bougainville, Fleurieu, Lacepède et Cuvier, fit l'éloge du travail de Péron et Lesueur saluant le dévouement des deux voyageurs : 
"Tout ce qu'il était physiquement possible de conserver, ils l'ont rapporté, soit dans l'alcool, soit empaillé avec soin, soit desséché, soit dans l'eau surchargée de muriate de soude. En un mot, ils n'ont négligé aucun des moyens connus pour multiplier leurs collections et pour les rendre aussi belles que possible. Plus que tous leurs prédécesseurs, MM. Péron et Lesueur ont rapporté des collections riches et nombreuses, et cependant ils n'ont rien voulu réserver pour eux-mêmes ; procédé d'autant plus généreux, qu'ils n'avoient point de modèles en ce genre parmi leurs prédécesseurs $\|^{54}$.

Le naturaliste et l'artiste ${ }^{55}$, seuls rescapés des cinq zoologistes originalement nommés ${ }^{56}$, permirent d'accumuler une collection zoologique :

«[...] dont chaque jour dévoile mieux l'étendue et l'importance. Plus de cent mille échantillons d'animaux d'espèces grandes et petites la composent : elle a déjà fourni plusieurs genres importants [...] et le nombre des espèces nouvelles, d'après le rapport des professeurs $d u$ Muséum, s'élève à plus de deux mille cinq cents $»^{57}$.

Que ne furent pas meilleure garantie pour le lithocolle que le salut de ces scientifiques de renom et la richesse des spécimens rapportés au Muséum. Péron fut si attaché à ses collections que dans un de ses rares portraits réalisé quinze jours avant sa mort, son ami et compagnon de voyage Charles Lesueur, le représenta assis de profil, lisant dans son intérieur, où carte, ouvrages, plume et encrier, et instruments de navigation rappelèrent ses activités de voyageur-naturaliste, et où cinq fioles lutées posées discrètement sur le coin de la cheminée fixèrent dans la mémoire collective le naturaliste à son invention et à ses collections. 
Figure 7. «F. Péron. Il s'est desséché comme un arbre chargé des plus beaux fruits qui succombe à l'excès de sa fécondité ». PERON François et FREYCINET Louis, Voyage de découvertes aux terres australes, Vol.II, Paris, Imprimerie royale, 1816.

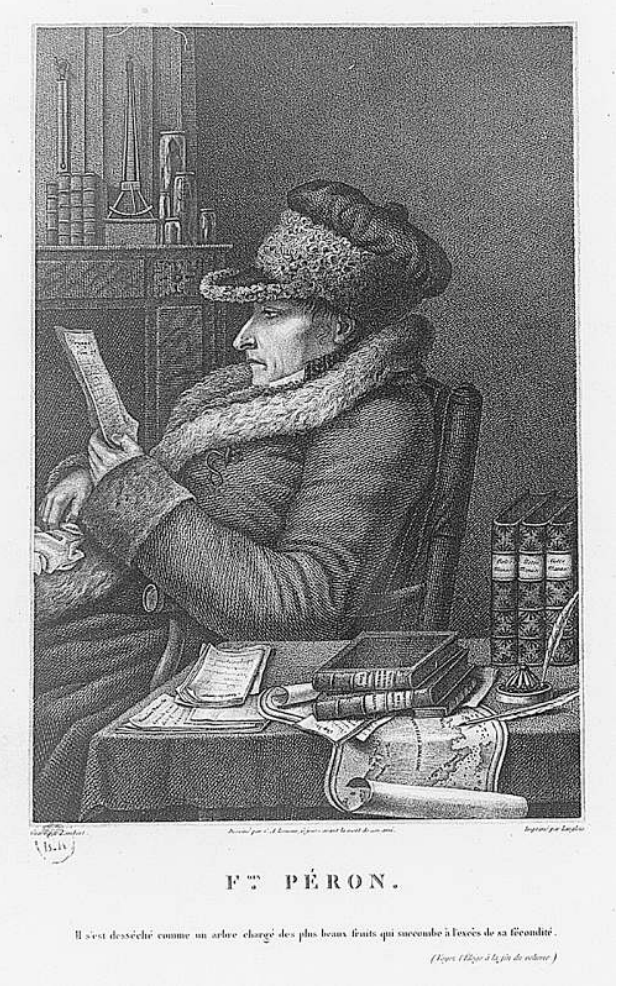

Portrait de François Péron, naturaliste-voyageur, lisant dans son intérieur, où carte, ouvrages, plume et encrier, et instruments de navigation illustrent ses activités de voyageur-naturaliste. On distingue cinq fioles lutées posées discrètement sur le coin de la cheminée illustrant à la fois la quantité importante de collections rapportées au Muséum d’histoire naturelle de Paris, et son invention du lithocolle.

(c) Droits réservés - Source gallica.bnf.fr / Bibliothèque nationale de France.

\section{Les émules du lithocolle}

Les faits validèrent la méthode mise en œuvre par Péron, et confirmèrent l'usage du lithocolle. Très loin d'une expérimentation devant une audience en pamoison ou d'une science en spectacle ${ }^{58}$, le lithocolle allait subir «les usages sociaux de la preuve ${ }^{59}$ » et s'imposer grâce à différents réseaux d'influences.

Le premier d'entre eux fut celui du Muséum national d'histoire naturelle. Visiblement, les retours d'exploration et le désœuvrement de certains naturalistes obligèrent à penser bien en amont les expéditions dans leur globalité : leur organisation, l'acquisition des connaissances naturalistes de la zone, la préparation et la formation des personnes, la pertinence du matériel nécessaire à la préparation. Sur invitation du ministre de la Marine, les professeurs de l'établissement livrèrent « des ordres aux chefs des colonies et aux commandants des vaisseaux de l'Etat pour qu'ils se procurent dans les divers pays où ils séjourneront les objets qui manquent au Muséum ". Cette Instruction (1818) ${ }^{60}$ comprenait notamment, au premier titre, "la manière de recueillir et de préparer les objets d'histoire naturelle ", suivie de « la manière de les emballer et de les faire parvenir en France dans le meilleur état possible ». Concernant la parfaite conservation des animaux dans la liqueur, les professeurs reprirent le Mémoire de Péron : 


\begin{abstract}
«[...] on sait que ce naturaliste auquel le Cabinet du Roi doit la plus riche collection d'animaux invertébrés, avait réussi à les conserver parfaitement. C'est après beaucoup de recherches et d'expériences qu'il était parvenu à découvrir les moyens les plus simples et les plus faciles $»^{61}$
\end{abstract} peuvent, sans inconvénient, être renversés sens dessus dessous, être exposés à toutes les secousses de la tempête, et supporter les plus fortes chaleurs, sans que l'alcool puisse s'échapper ${ }^{62}$ ». La même année, était décidée, sur la proposition du ministre de l'intérieur, la création d'une école de voyageurs-naturalistes, avec une dotation de 20000 francs. Placée sous la direction des professeurs du Muséum, elle ouvrit ses portes le 10 février $1819^{63}$. Les élèves étaient choisis sur concours et après avoir reçu une instruction suffisante, en général d'un an, ils étaient envoyés dans les différentes parties du monde aux frais et pour l'État. Au cours du XIXe siècle, les voyages naturalistes se démultipliant, l'Instruction aux voyageurs connut cinq rééditions (1824, 1827, 1829, 1845, 1860). Par le biais de sa formation dispensée, des différentes publications et de sa politique active de missions naturalistes, le Muséum participa à l'uniformisation et à la normalisation progressive de la collecte et de la préparation des collections. Le choix du lithocolle (composition, préparation, application) fut ainsi recommandé à la nouvelle génération de voyageurs-naturalistes, tel Auguste Plée (1786-1825), formé à l'école du Muséum, qui fait remarquer que :

«Poissons dépouillés, tous les animaux placés dans la liqueur, sont très bien conservés et les trois cent cinquante bocaux sont lutés comme indiqué par le Muséum auquel cependant j'ai ajouté quelque chose qui m'a paru utile : c'est un parchemin en dessous du bouchon et un autre parchemin recouvrant le lithocolle, ce qui empêche toute évaporation du liquide $[. ..] »^{64}$.

37

Ces naturalistes formés au Muséum alimentèrent un second réseau d'influence, celui des naturalistes-voyageurs souvent appelés à collaborer dans la rédaction d'ouvrages généralistes tels que des Annales ou des Dictionnaires, abondants au XIXe siècle. Ainsi, Charles-Louis Bourdet de la Nièvre, naturaliste-voyageur et correspondant de plusieurs académies et sociétés savantes, partagea avec les lecteurs, un extrait d'une lettre de Péron revenu depuis peu en France (1805) au sujet des luts :

«Les procédés dont vous vous êtes servies, sont ceux qu'on nous avait recommandés à notre départ; ces moyens s'étant trouvés en défaut, il nous a fallu créer un nouveau système de fermeture et qui a réussi au-delà même de nos espérances " $^{65}$.

Bourdet révéla que Péron lui aurait personnellement divulgué le procédé du lithocolle cinq ans avant sa publication dans le Journal de Physique. En août 1819, le naturaliste René Primevère Lesson (1794-1849) dédia un article sur les méthodes de conservation dans les Annales maritimes ${ }^{66}$ et emprunta également l'article de Péron du Journal de Physique (1810). Rompu aux conditions particulières des voyages-naturalistes ${ }^{67}$, Lesson fut une personne déterminante dans la médiatisation du lithocolle, grâce à ses nombreux articles, entre autres sur la taxidermie dans le Dictionnaire des sciences naturelles $(1828)^{68}$ dans lequel il

e-Phaïstos, VII-1 | 2019 
dédia le troisième chapitre à la «Fermeture et couverture des vases " et reprit les méthodes du naturaliste Péron qui «ayant reconnu l'insuffisance de plusieurs, en a substitué d'autres plus favorables et dont un voyage lointain a grandement prouvé la bonté et les avantages " ${ }^{69}$, ou encore celui portant sur les " Préparations conservatrices » dans le Dictionnaire classique d'histoire naturelle $(1828)^{70}$. Les naturalistes-préparateurs vinrent également apporter leur sceau, les plus connus et reconnus relayèrent son usage dans les manuels de préparateur ${ }^{71}$. Ce faisant, le lithocolle investit les écoles de médecine ou de pharmacie pour la préparation des collections ${ }^{72}$. Le procédé fut aussi cité dans divers dictionnaires des inventions ${ }^{73}$ qui selon les références, scellèrent ses inventeurs, Péron et Lesueur, à la date erronée de 1811. Bien entendu malgré sa popularité, le lithocolle trouva des détracteurs comme le créationniste Hercule Straus-Durckheim (1790-1865), auteur de la Théologie de la Nature (1852), qui critiqua Péron et Lesueur :

\footnotetext{
«Les auteurs n'indiquent point les proportions, et moi-même je n'ai jamais fait usage de lithocolle pour luter les bocaux; mais je l'ai souvent vu employer, et ne lui trouve d'autre avantage que de sécher rapidement ; mais comme les matières résineuses qui le composent sont solubles dans l'alcool, ce lut ne ferme que fort mal les bocaux contenant ce liquide $»^{74}$.
}

Preuve néanmoins de son efficacité, le lithocolle de Péron dépassa très rapidement les frontières de l'hexagone pour être recommandé par exemple aux voyageurs-naturalistes anglo-saxons ${ }^{75}$. Le Royaume-Uni adopta le lithocolle qui fut cité régulièrement dans de nombreux ouvrages de taxidermie au cours du XIX ${ }^{e}$ siècle ${ }^{76}$. Deux britanniques lui donnèrent ses lettres de noblesse. Thomas Brown (1789-1855), ornithologue de renom pour ses Instructions for Collecting and Preserving Subjects of Natural History (1808), le mentionna dès la première édition de son ouvrage The Taxidermist's manual (1833) et dans la vingtaine qui suivraient ${ }^{77}$. Montagu Browne (1837-1923), directeur du Muséum de Leicester et figure de l'histoire de la taxidermie britannique, loua son efficacité dans ses ouvrages de références de taxidermie ${ }^{78}$. A la fin du XIX siècle, le lithocolle fit également une incursion en Allemagne, en chirurgie orthopédique comme ciment biologique pour fixer des fractures osseuses ${ }^{79}$.

\section{Le Mastic au caoutchouc de Maissiait (1847) et le Ciment-Lut de Deyrolles (1903)}

A partir du milieu du XIX ${ }^{e}$ siècle, les lutages traditionnels se complexifièrent à mesure que les matériaux le composant se diversifièrent, employant des matières naturelles jusque-là non testées, comme par exemple le caoutchouc. En séance du lundi 8 mars 1847, Jacques Maissiat, professeur agrégé de la Faculté de Médecine de Paris, lut devant l'Académie des sciences une note Sur un moyen de fermer exactement les vases destinés aux collections d'histoire naturelle ${ }^{80}$, également applicable à la conservation des substances alimentaires et des objets d'anatomie comparée exposés et conservés dans les liqueurs. En présence des Commissaires désignés, le chimiste Jean-Baptiste Dumas (1800-1884), et les Professeurs du Muséum de Paris, Henri Milne Edwards (1800-1885) et Achille Valenciennes (1794-1865), Maissiat rappela les efforts et les recherches sérieuses des naturalistes Glauber, Réaumur et Daubenton. Le nouveau lut -le Mastic unissant- se composa de caoutchouc dissous et combiné à de la chaux, présenta les avantages d'être 
sans odeur, insoluble dans l'eau et l'alcool, persistant durant des années, et très plastique. Furent soumis comme preuve aux membres, divers bocaux anatomiques ${ }^{81}$, dont un « un marteau d'eau ${ }^{82}$ fait avec l'alcool le plus concentré du commerce dans un vase ainsi fermé » et un bocal conservant des fruits, ouvert devant le comité constatant leur "parfait état de conservation ». Le lut de Maissiat fut ensuite exposé par Edmond Becquerel (1820-1891), physicien et aide-naturaliste du Muséum, devant la Société d'encouragement pour l'industrie nationale lors de la séance 22 juillet $1847^{83 .}$ Le conseil conquis lui décerna la médaille d'argent d'encouragement. Parallèlement conservateur du musée Orfila, Maissiait généralisa son lut aux collections anatomiques conservées en fluide du Musée Dupuytren, qui «rendu de grands services [car] tous les procédés antérieurs étant très défectueux et d'un emploi difficile ${ }^{84}$. Ce lut fut popularisé sous le nom de mastic au caoutchouc de Maissiat ${ }^{85}$ jusque dans les années 1930, puis commercialisé par le joint «Le Parfait » (1930), expliquant de nos jours la présence pléthorique de ces joints orangés dans les collections en fluide. Le caoutchouc remplaça bon nombre d'anciens lutages lorsque ces derniers devaient être rénovés ou se généralisa dans la réalisation des nouveaux luts. En 1910, le Professeur Léon Vaillant révélait que le lut des collections d'Herpétologie du Muséum se présentait en un millefeuille de mastic de vitrier couvert d'une peau de vessie de porc collée avec de la gomme arabique, et recouverte d'une lamelle de papier d'étain :

\footnotetext{
«Cette méthode, excellente du point de vue du résultat comme on peut s'en assurer sur les bocaux restant de l'ancienne collection, présente l'inconvénient d'être longue à élaborer et de manipuler les bocaux à plusieurs reprises pour laisser le mastic séché en veillant à ce que l'alcool ne le touche pas $»^{86}$.
}

Vaillant admit que cet ancien mastic dû être remplacé par celui à base de caoutchouc. Mais pour éviter les risques liés à l'emploi d'un bec de gaz en présence des collections en alcool pour le ramollir, le mastic en caoutchouc fut à son tour remplacé par un lutage élaboré par Angel, préparateur du laboratoire d'Herpétologie, constitué d'un kilogramme de mastic vitrier, de $100 \mathrm{~g}$ de litharge (oxyde de plomb), de noir d'ivoire ${ }^{87}$ et de brun rouge (oxyde fer) maintenant la couleur originelle du caoutchouc au lut nouveau. A partir du milieu du XIXe, la communauté des naturalistes amateurs augmenta ; la pratique de la collecte se popularisa et en conséquence une demande de matériels nécessaire à la constitution collection et de conseils s'est accrût donnant naissance à de nombreux magasins naturalistes ${ }^{88}$. Le plus connu d'entre eux, l'établissement parisien Deyrolle proposa dès 1893 son Ciment-Lut " donnant une fermeture hermétique et immédiate » De composition inconnue, il était encore présent dans les années 1930 dans le catalogue de vente ${ }^{90}$, et dans des encarts publicitaires de la revue Le Naturaliste, édité par la maison Deyrolle, « l'alcool reste des années sans montrer la moindre évaporation. Les résultats sont surprenants ». 
Figure 8. Encart publicitaire et mode d'emploi du Ciment-Lut développé par l'établissement parisien Deyrolle dans journal Le Naturaliste. Numéro $1^{\mathrm{er}}$ novembre 1893, Le Naturaliste, Paris, Bureaux du journal, 1893.

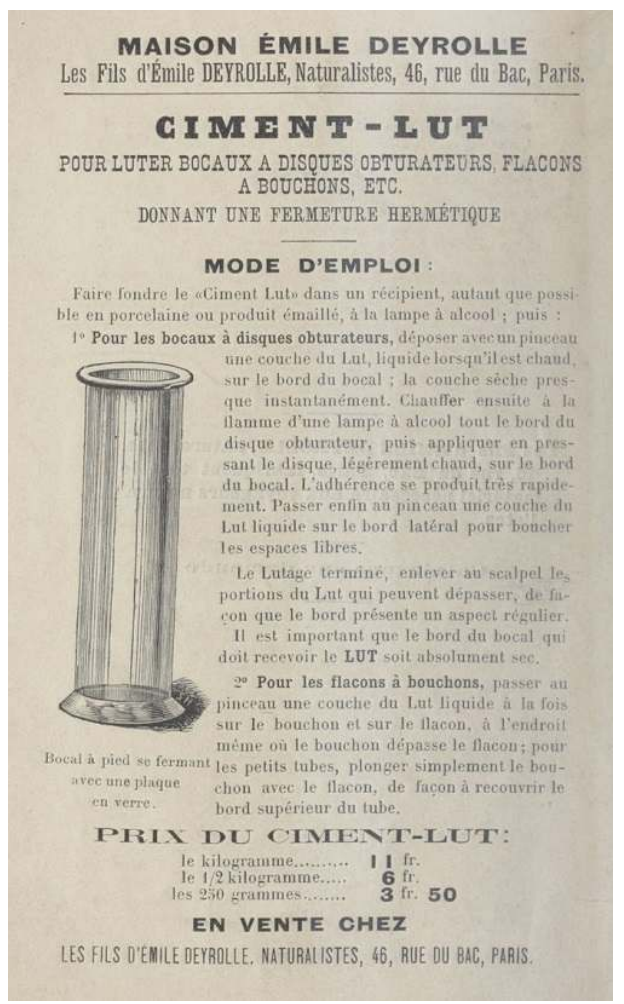

Créée en 1831 par Jean-Baptiste Deyrolle, entomologiste et passionné d'histoire naturelle, la boutique naturaliste vend essentiellement des insectes, du matériel naturaliste, et quelques taxidermies. Lui succédèrent son fils Achille, puis son petit-fils Emile qui développa l'entreprise en élargissant le commerce au matériel d'enseignement (tableaux muraux en couleurs, modèles anatomiques, pièces biologiques), en ajoutant une section Physique et Chimie, et en créant le journal Le Naturaliste (1 ${ }^{\text {er }}$ avril 1879). En 1880, son arrière-petit-fils Gabriel et le gendre de ce dernier Paul Groult-Deyrolle, se chargèrent de la direction. Les agrandissements de la Samaritaine expulsèrent en 1890 l'entreprise, et Deyrolle s'installa alors à l'adresse mythique du 46 rue du Bac, où l'établissement réside toujours actuellement.

(c) Droits réservés -Source gallica.bnf.fr / Bibliothèque nationale de France

A l'instar de l'usage du savon arsenical dans le tannage des spécimens naturalisés ou du mercure dans les herbiers en France ${ }^{91}$, le lithocolle de Péron ou le mastic de caoutchouc de Maissait sont des marqueurs chrono-typologiques dans l'histoire des préparations des collections conservées en fluide. Porteur de ces marqueurs historiques, l'objet revêt alors une valeur testimoniale pour l'histoire collections et des techniques associées ${ }^{92}$. Daubenton ne se berça pas d'illusion sur le lutage, «[o]n ne doit espérer d'empêcher absolument l'évaporation par toutes ces pratiques, tout ce que l'on peut faire par-là, est de la retarder et d'en diminuer la quantité $»^{93}$. De nos jours, le lutage demeure un enjeu pour assurer la pérennité des collections et posent de fait un questionnement axiologique entre l'usage scientifique du spécimen et la valeur historique de l'objet ${ }^{94}:$ les maintenir dans un culte d'ancienneté malgré qu'ils puissent mettre en péril la conservation d'un organisme, les restaurer pour garder en mémoire ce témoignage, les détruire ou les substituer totalement car l'usage le nécessite quitte à perdre sa valeur d'historicité. 


\section{NOTES}

1. Voir, entre autres, pour les voyages-naturalistes : LAISSUS Yves, «Les voyageurs naturalistes du Jardin du roi et du Muséum d'histoire naturelle : essai de portrait-robot », Revue d'histoire des sciences, 1981, p.259-317 ; DROUIN, Jean-Marc, « De Linné à Darwin : les voyageurs naturalistes » dans SERRES Michel (dir.), Eléments d'histoire des sciences, Paris, Bordas, 1989, p. 321-335 ; COLLINI Silvia et VANNONI Antonella, Les instructions scientifiques pour les voyageurs (XVII ${ }^{e}-\mathrm{XIX}^{\mathrm{e}}$ ), Paris, Éditions L'Harmattan, 2005 ; LINON-CHIPON Sophie et VAJ Daniela, Relations savantes: voyages et discours scientifiques, Presses Paris Sorbonne, 2006 ; NEMO Jean, Présences françaises outre-mer (XVI ${ }^{e}$ $\mathrm{XXI}$ siècles), II - Science, religion et culture, Paris, Académie des sciences de l'outre-mer, Karthala, 2012 ; CHAPPEY Jean-Luc et DONATO Maria Pia, «Voyages et mutations des savoirs. Entre dynamiques scientifiques et transformations politiques. Fin XVIII ${ }^{\mathrm{e}}$-début XIX ${ }^{\mathrm{e}}$ siècle », Annales historiques de la Révolution française, 3, 385, 2016, p.3-22 ; Voir pour les cabinets : POMIAN Krysztof, Collectionneurs, amateurs et curieux, Paris et Venise: XVe-XVIII siècles, Paris, Gallimard, 1987; SCHNAPPER Antoine, Le géant, la licorne, la tulipe, Paris, Flammarion, 1988 ; DAVENNE Christine, Modernité du cabinet de curiosités, Paris, Éditions L'Harmattan, 2004 ; LESTRINGANT Frank, Le théâtre de la curiosité, Paris, Presses Paris Sorbonne, 2008; LACOUR Pierre-Yves, La République naturaliste : collections d'histoire naturelle et Révolution française (1789-1804), Paris, Muséum national d'histoire naturelle, 2014 ; BONDAZ Julien, DIAS Nélia et JARRASSE Dominique, «Collectionner par-delà nature et culture ", Gradhiva, 1, 2016, p.28-49 ; LACOUR Pierre-Yves, "Coquilles et médailles. Naturalia et artificialia dans les collections de province autour de la Révolution", Gradhiva, 1, 2016, p.50-67 ; PELUCCHI Stéphane, «La collection du cabinet d'histoire naturelle de Lavoisier: Sa place dans son œuvre scientifique", Revue d'histoire des sciences, 69, 1, 2016, p.153-169; RUSQUE Dorothée, «Faire circuler les objets naturalistes au XVIII ${ }^{\mathrm{e}}$ siècle. Jean Hermann comme intermédiaire dans les échanges entre la France méridionale et l'espace germanique ", Liame [en ligne], 26, 2016, disponible sur URL : <http://liame.revues.org/568> (consultée le 20 mai 2017).

2. Dictionnaire de l'Académie française, première édition, Paris, Institut de France, 1694, p.671.

3. MALLET abbé Edmé-François, «Lut \& Luter» dans DIDEROT Denis et D’ALEMBERT Jean [Le Rond], Encyclopédie, ou Dictionnaire Raisonné des Sciences, des Arts et des Métiers, tome 9, Ju-Mamira, première édition, 1765, p. 754-756.

4. "Lut », selon le Dictionnaire de l'Académie Françoise, $4^{\mathrm{e}}$ édition, Paris, Chez la Vve B. Brunet, 1762, « C'est parmi les Chimistes, De la terre grasse, ou un mélange de blanc d'œuf \& de chaux, dont ils se servent pour boucher \& pour joindre les vases qu'ils mettent au feu ».

5. La colle de farine s'obtient en délayant à froid de la farine de froment dans huit fois et demi son poids d'eau. Le mélange est porté à ébullition pendant environ trois quarts d'heure et agitée. Lorsque la colle file, elle est transférée dans un récipient en terre, puis passer dans un tamis avant qu'elle refroidisse.

6. LEBEAUD Nicolas et JULIA DE FONTENELLE Jean, Manuel complet théorique et pratique du distillateur et du liquoriste, ou Traité de la distillation : contenant l'art de fabriquer les diverses espèces d'eau-de-vie et esprits..., Paris, Roret, 1826 (Première édition), 1835 (Quatrième édition), p.112-116.

7. Revue Encyclopédique ou analyse raisonnée des productions les plus remarquables dans la littérature, les sciences et les arts / par une réunion de membres de l'Institut et d'autres hommes de lettres, T.XXIX, Paris, Bureau Central de la Revue Encyclopédique, 1826, p.206. 
8. Voir les descriptions des cabinets d'histoire naturelle réalisées par DESALLIER D'ARGENVILLE Antoine-Joseph, La conchyliologie, ou histoire naturelle des coquilles de mer, d'eau douce, terrestres et fossiles : avec un traité de la zoomorphose, ou représentation des animaux qui les habitent: ouvrages dans lequel on trouve une nouvelle méthode de les diviser, I, Paris, Guillaume de Bure fils aîné, 1780.

9. GERSAINT Edme-François, Catalogue raisonné d'une collection de diverses curiosités en tous genres: contenus dans les cabinets de feu Monsieur Bonnier de la Mosson, Paris, Jacques Barois \& Pierre Guillaume Simon, 1744 .

10. DAUBENTON Louis-Jean-Marie, "Description du Cabinet du Roy", Histoire Naturelle des Animaux Quadrupèdes, IV-XV, Paris, Imprimerie nationale, 1749. Daubenton n'a pas poursuivi sa description pour les tomes suivants. A partir des mentions faites par Daubenton pour chaque objet présent dans sa Description, nous avons réalisé la répartition des spécimens Quadrupèdes de la collection du Cabinet du Roy, selon leur mode de préparation, a été réalisée.

11. Esprit est l'ancien terme pour définir divers gaz ou produits liquides et volatils: Esprit alcalin, ammoniac; Esprit-de-vin, alcool éthylique obtenu par distillation du vin; Esprit-de-bois, alcool méthylique ;Esprit-de-sel, acide chlorhydrique.

12. "Sur la manière d'empêcher l'évaporation des liqueurs spiritueuses, dans lesquelles on conserve des pièces d'histoire naturelle", Collection académique composée des mémoires, actes ou journaux des plus célèbres académies \& sociétés littéraires de l'Europe: concernant la physique, l'histoire naturelle, la botanique, la chimie, l'anatomie, la médecine, la mécanique, X, 1785, p. 11-12 (p.17-22).

13. DAUBENTON, Louis-Jean-Marie, L'Histoire Naturelle générale et particulière : avec la Description du Cabinet du Roy, III, Paris, Imprimerie Royale, 1749, p. 176-195.

14. DAUBENTON, Louis-Jean-Marie, «Sur les moyens de conserver les quadrupèdes ovipares, et d'autres animaux, après la mort ", Encyclopédie méthodique ou par ordre de matières. Histoire naturelle des oiseaux, Paris, Plomteux, II, 1784, p.564-570.

15. DAUBENTON, Louis-Jean-Marie, L'Histoire Naturelle générale et particulière : avec la Description du Cabinet du Roy, III, Paris, Imprimerie Royale, 1749, p.181.

16. GLAUBER Johann Rudolph, La description des nouveaux fourneaux philosophiques, ou Art distillatoire, Partie I, Paris, Thomas Jolly, 1659, p.13-15.

17. "Sur la manière d'empêcher l'évaporation des liqueurs spiritueuses, dans lesquelles on conserve des pièces d'histoire naturelle", Collection académique composée des mémoires, actes ou journaux des plus célèbres académies \& sociétés littéraires de l'Europe: concernant la physique, l'histoire naturelle, la botanique, la chimie, l'anatomie, la médecine, la mécanique, X, 1785, p.12.

18. Ibidem, p.11-14; REAUMUR [FERCHAULT DE] René-Antoine, "Moyens d'empêcher l'évaporation des liqueurs spiritueuses, dans lesquelles on veut conserver des productions de la Nature de différents genres », Histoire de l'Académie Royale des Sciences, Paris, Imprimerie royale, 1751, p.483-516 ; «Addition au Mémoire sur les manières d'empêcher l'évaporation des liqueurs spiritueuses renfermées dans des bocaux", Histoire de l'Académie Royale des Sciences, Paris, Imprimerie royale, 1751, p.516-539.

19. Ibidem, p.517.

20. DAUBENTON Louis-Jean-Marie, « Sur les moyens... », op.cit., 1784, p.567.

21. Le Blanc de Troyes est un pigment à base de poudre de craie extraite dans la région de Troyes. Il fut utilisé comme pigment dans les peintures, agent blanchissant par les drapiers, et il entra dans la composition de certains papiers et cartons, et de mastic de vitriers.

22. Composé de trois cinquièmes de mercure et de deux cinquièmes de plomb fondu ensemble.

23. DAUBENTON Louis-Jean-Marie, « Sur les moyens... », op.cit., 1784, p.570.

24. VICQ D'AZYR Félix, LINDET Thomas et BOUQUIER Ainé, Instruction Sur la manière d'inventorier et de conserver, dans toute l'étendue de la République, tous les objets qui peuvent servir aux arts, aux sciences et à l'enseignement, proposée par la Commission temporaire des 
arts, et adopté par le Comité d'instruction publique de la Convention nationale, Paris, Imprimerie nationale, 1793, p.16-17.

25. LESSON René-Primevère, "Préparations conservatrices », Dictionnaire classique d'histoire naturelle, XIV, Paris, Rey et Gravier, \& Baudouin Frères, 1828, p.266-273.

26. Mélange de gomme-laque ou de résine et d'essence de térébenthine, de suif, et de pigments, cette cire fut plus connue comme cire à cacheter les lettres ou les bouteilles. La cire d'Espagne aurait été inventée, sous le règne de Louis XIII, par un sieur Rousseau à Perpignan qui à l'époque appartenait à l'Espagne.

27. HENDRIKSEN MARIEKE, Elegant Anatomy: The Eighteenth-Century Leiden Anatomical Collections, Leyde, Brill, 2014.

28. VAN DE ROEMER Gijsbert M., " From vanitas to veneration: The embellishments in the anatomical cabinet of Frederik Ruysch », Journal of the History of Collections, 22, 2, 2009, p.169-186.

29. MULDER Wim, «How to prepare an anatomical specimen? ", 2009, International Ruyschresearchgroup [en ligne] http://ruysch.dpc.uba.uva.nl/cgi/t/text/text-idx?page=ruyschhowto;c=ruysch;cc=ruysch;lang=en (consultée le 27 juin 2017).

30. MARTIN Pierre, " Portrait du Dr. Ruysch en boucher subtile ", dans BouTEILLE-MESITER Charlotte et AUKRUST Kjerstin, Corps sanglants, souffrants et macabres: XVI ${ }^{e}$ XVII ${ }^{e}$ siècle, Paris, Presses Sorbonne Nouvelle, 2010, p.50-51.

31. HANSEN Julie V., « Resurrecting death: anatomical art in the cabinet of Dr. Frederik Ruysch ", The Art Bulletin, 78, 4, 1996, p.663-680.

32. KNOEFF Rina, "Touching anatomy: On the handling of preparations in the anatomical cabinets of Frederik Ruysch (1638-1731) ", Studies in History and Philosophy of Science Part C: Studies in History and Philosophy of Biological and Biomedical Sciences, 49, 2015, p.32-44.

33. Ibidem, p. 37.

34. Portrait de Seba, réalisé par le graveur Jakob Houbraken (1698-1780) d'après une peinture de Jan Maurits Quinkhard (1688-1772).

35. JEANNIN Camille, Le Thesaurus du cabinet d'Albertus Seba interprété par Antoine-Jean Coquebert de Montbret, Master 2 «Culture de l'écrit et de l'image », Université Lumière Lyon 2, 2016, [en ligne] disponible sur <http://www.enssib.fr/bibliotheque-numerique/ documents/67130-le-thesaurus-du-cabinet-d-albertus-seba-interprete-par-antoine-jean-

coquebert-de-montbret.pdf > (consultée le 6 février 2017).

36. WINTROUB Michael, « Taking stock at the end of the world: Rites of distinction and practices of collecting in early modern Europe ", Studies in History and Philosophy of Science, vol.31, n³, 1999, p.395-424.

37. TURGOT Etienne-François, Mémoire instructif sur la manière de rassembler, de préparer, de conserver, et d'envoyer les diverses curiosités d'histoire naturelle; auquel on a joint un mémoire intitulé: Avis pour le transport par mer, des arbres, des plantes vivaces, des semences, \& de diverses autres curiosités d'histoire naturelle, Paris, Bruysset, 1758, p. iij.

38. LETTSOM John Coakley, Les voyageur naturaliste, ou Instructions sur les moyens de ramasser les objets d'histoire naturelle, et de bien les conserver, Paris \& Amsterdam, Lacombe, 1775, p.viij.

39. KUCKHAN Tesser Samuel, «Four letters from Mr. TS Kuckhan [sic] to the President and Members of the Royal Society, on the preservation of dead birds ", Philosophical Transactions, 60, 1770, p.302-320. Cette publication sera traduite et publiée en France, KUCKHAN Tesser Samuel "Quatre lettres sur la manière d'embaumer les oiseaux », Journal de Physique, IV, 1773, p.147-154.

40. KURY Lorelai, "Les instructions de voyage dans les expéditions scientifiques françaises (1750-1830) », Revue d'histoire des sciences, vol.51, n¹ (1998), p.65-91.

41. Péron et Lesueur participèrent à la grande expédition (1800-1803) conduite par Nicolas Baudin, à bords des navires le Géographe et le Naturaliste dans les mers du sud. Voir JANGOUX Michel, «Les zoologistes et botanistes qui accompagnèrent le capitaine Baudin aux Terres 
australes », Australian Journal of French Studies, 4, 2, 2004, p.55-78 ; FORNASIERO Jean et WESTSOOBY John, «Voyages et déplacements des savoirs. Les expéditions de Nicolas Baudin entre Révolution et Empire ", Annales historiques de la Révolution française, 385, 3, 2016, p.23-46.

42. PERON François et LESUEUR Charles-Alexandre, " Mémoire sur la conservation des diverses espèces d'animaux dans l'alcool », Journal de Physique, LXXXI, 1810, p.265-288.

43. Bien que l'article fût co-signé par Péron et Lesueur et que l'usage du pronom personnel de la première personne du pluriel soit effectif, Péron semble avoir été l'auteur principal. En effet, dans la publication du second volume du Voyages de découvertes aux terres australes (1816), Louis Freycinet dédie le chapitre XXXIX au Fragment $d u$ « Mémoire de MM. Péron et Lesueur sur l'Art de conserver les animaux dans les collections zoologiques»(p.373-392). En première page, l'auteur signale en note "Malgré les recherches que M. Lesueur et moi avons faites dans les papiers de M. Péron, il ne nous pas a été possible de retrouver la totalité de ce mémoire, dont il manque 8 pages au commencement». Les parties publiées proviennent d'un «brouillon informe » dont de nombreuses lacunes sont notifiés par l'auteur. Freycinet clôture le chapitre : « Nous devons regretter sans doute ne pouvoir donner la suite d'un travail aussi utile; mais il n'a jamais été achevé ; et cette privation ajoute encore aux regrets que doit inspirer la mort de Péron à tous les véritables amis des sciences naturelles ".

44. Voir LAISSUs Yves » Un naturaliste exemplaire, François Péron (1775-1810) », Bulletin de la Société d'émulation du Bourbonnais, 2e trimestre, Moulins, 1976, p. 70.

45. Antoine-François de Foucroy (1755-1809) a été professeur de chimie en 1784 par Buffon au Jardin royal des plantes médicinales, puis devient en 1793 le premier titulaire de la chaire de Chimie générale au Muséum avec Antoine-Louis Brongniart pour démonstrateur. Il assurera la direction du Muséum pour les périodes 1800-1801 et 1804-1805. Voir JAUSSAUD Philippe et BRYGOO Edouard-Raoul, Du Jardin au Muséum en 516 biographies, collection Archives, Paris, Muséum national d'Histoire naturelle, 2004.

46. Il fait mention de deux sortes de brai, tous deux tirés originalement des pins et sapins. Le brai sec est la matière résineuse qui a été cuite avec un mélange d'eau, devenue une matière sèche et cassante, transparente et de couleur roussâtre/noirâtre. Le brai gras est, quant à lui, un mélange de brai sec et des matières grasses (goudron, suif) qui fondus ensemble donnent une masse liquide. Dans les navires, le brai est employé pour le calfatage : le brai sec pour les coutures du fonds et le brai gras pour celle du haut.

47. PERON François et LESUEUR Charles-Alexandre, « Mémoire... », op.cit., 1810, p.269.

48. Ibidem, p.271.

49. Pour Péron, « lithocolle » est un nom masculin, par extension du terme mastic lithocolle. Le Dictionnaire universel (1712) définit la lithocolle -nom féminin- comme étant un ciment employé par les lapidaires pour maintenir les pierres lorsqu'elles sont taillées ou polies sous une meule. Ce ciment était composé de poix (résine et goudrons végétaux) et de brique. Employée comme mortier, la lithocolle contenait de la poudre de marbre et de la colle forte. Utilisée pour coller des éclats de pierre, elle était alors un mélange de blanc d'œuf et de poix.

50. PERON François et LESUEUR Charles-Alexandre, « Mémoire... », op.cit., 1810, p.274.

51. Ibidem, p.275.

52. Ibid., p.275-276.

53. Ibid., p.276.

54. Ibid., p.VIII.

55. VIAL Danièle, «Les méduses sur vélins de C.-A. Lesueur: valeur taxinomique d'une œuvre d'art au XIX siècle (1800-1810) », Bulletin de la société d'histoire et d'épistémologie des sciences de la vie , 23, 2, 2016, p.135-153.

56. Jean-Baptiste Bory de Saint-Vincent, officier d'état-major, zoologiste en chef, et Désiré Dumont, zoologiste adjoint à bord du Naturaliste, quittèrent l'expédition à l'île de France en mars 1801. Stanislas Levillain et René Maugé de Cely, zoologistes à bord du Géographe, moururent de 
dysenterie au large de Timor le 23 décembre 1801 pour le premier, et au large de la Tasmanie, le 21 février 1802, pour le second.

57. «Rapport fait au gouvernement par l'Institut Impérial sur les voyages de découvertes aux Terres Australes ", Extrait du Procès-verbal de la Classe des Sciences physiques et mathématiques, séance du lundi 9 juin 1806, dans PERON François, Voyage de Découvertes aux Terres Australes, I, Paris, Arthus Bertrand, 1807, p.II.

58. Voir SCHAFFER Simon, "Natural Philosophy and Public Spectacle in the Eighteenth Century ", History of science, vol.21, $\mathrm{N}^{\circ} 1,1983$, p.1-43; BENSAUDE-VINCENT Bernadette et BLONDEL Christine (dir). Science and Spectacle in the European Enlightenment, Ashgate, Aldershot, 2008 ; NIETO-GALA Agustí Science in the Public Sphere: A History of Lay Knowledge and Expertise, London et New York, Routledge, 2016.

59. RUELLET Aurélien, «Les stratégies de l'expérience ", Tracés. Revue de Sciences humaines, $\mathrm{n}^{\circ} 7$, 2004, p.61-75.

60. Il existe deux publications du même texte : «Instruction. Sur les recherches qui pourraient être faites fans les colonies, sur les objets qu'il serait possible d'y recueillir, et sur la manière de les conserver et de les transporter ", Mémoires du Muséum d'histoire naturelle par les professeurs de cet établissement, IV, 1818, p.193-239; Instruction. Sur les recherches qui pourraient être faites fans les colonies, sur les objets qu'il serait possible d'y recueillir, et sur la manière de les conserver et de les transporter, Paris, Belin, 1818.

61. Mémoires du Muséum d'histoire naturelle par les professeurs de cet établissement, IV, 1818, p.209.

62. Ibidem, p. 213.

63. Archives du Muséum national d'histoire naturelle, AJ15/865.

64. THESEE Françoise, Auguste Plée, 1786-1825, un voyageur naturaliste : ses travaux et ses tribulations aux Antilles, au Canada, en Colombie, Paris, L'Harmattan, 1989, p.161.

65. BOURDET Charles-Louis, Mémoire à Messieurs les professeurs administrateurs du Muséum d'Histoire naturelle au jardin du roi : sur les qualités et les connaissances que doit avoir un naturaliste voyageur, Liège, Walthard et Burgdofer, p.10

66. LESSON René-Primevère, « De la conservation des objets d'histoire naturelle par des liquides spiritueux, salins, acides, etc. », Annales maritimes et coloniales, IIe partie, Paris, Imprimerie royale, 1819, p.694-704.

67. Lesson a participé au voyage autour du monde à bord de La Coquille (1822) comme médecin et botaniste. Voir J. LESCURE, «René-Primevère LESSON (1794-1849), pharmacien de la Marine, voyageur-naturaliste et herpétologiste », Bulletin de Société Herpétologique de France, n¹55, 2015, p.1-50.

68. LESSON René-Primevère, §.3. Fermeture et couverture des vases, p.430-433 dans " De la conservation des objets d'histoire naturelle par des liquides spiritueux, salins, acides, etc.», « Taxidermie », Dictionnaire des Sciences Naturelles dans lequel on traite méthodiquement des différents êtres de la nature, considérés soit en eux-mêmes, d'après l'état actuel de nos connaissances, soit relativement à l'utilité qu'en peuvent retirer la médecine, l'agriculture, le commerce et les arts, suivi d'une biographie des plus célèbres naturalistes, LII, Strasbourg, Levrault, Paris, Le Normant, 1828, p.353-436.

69. Ibidem, p.433.

70. LESSON René-Primevère, "Préparations conservatrices", Dictionnaire classique d'histoire naturelle, t.XIV, Paris, Rey et Gravier, \& Baudouin Frères, 1828, p.266-273.

71. BOIRARD Pierre, Manuel du naturaliste préparateur, ou l'art d'empailler les animaux et de conserve les végétaux et les minéraux, Paris, Roret, 1825, p.178-180 ; BOIRARD Pierre, Nouveau manuel complet du naturaliste préparateur ou l'art d'empailler les animaux, de conserver les végétaux et les minéraux, de préparer les pièces d'anatomie normale et pathologique: suivi d'un traité des embaumements, Paris, Roret, 1852, p.286-287 ; BOIRARD Pierre, et CANIVET Emanuel, 
Manuel du naturaliste préparateur : ou, l'Art d'empailler les animaux et de conserver les végétaux et les minéraux, Paris, Roret, 1828, p.156-157 ; BOITARD Pierre et MAIGNE W., Nouveau manuel complet du naturaliste préparateur. Deuxième partie. Taxidermie, préparation des pièces anatomiques, contenant l'art d'empailler et de conserver les animaux vertébrés et invertébrés, de préparer les végétaux et les minéraux, Paris, Roret, 1890, p. 251-252 ; DUPONT M., Traité de taxidermie ou l'art de conserver et d'empailler les animaux, Paris, Mansut, 1827, p.100-101.

72. CHEVALLIER Alphonse, Dictionnaire des drogues simples et composées, ou Dictionnaire d'histoire naturelle médicale, de pharmacologie et de chimie pharmaceutique, III, 1828, p.434.

73. Dictionnaire chronologique et raisonné des découvertes, inventions, innovations, perfectionnements, observations nouvelles et importations en France, dans les sciences, la littérature, les arts, l'agriculture, le commerce et l'industrie de 1789 à la fin de 1820, Paris, Louis Colas, 1823, XI, p.165-166; BOQUILLON Nicolas, Dictionnaire des inventions et découvertes, depuis le commencement du monde jusqu'à nos jours, Paris, Audin, 1826, p.230; JOUFFROY Achille [Marquis de], Dictionnaire des inventions et découvertes anciennes et modernes, dans les sciences, les arts et l'industrie, volume II (H-Z), Petit Montrouge, abbé Migne, XXXVI, 1852-1853, p.392-393.

74. STRAUSS-DURCKEIM Hercule Eugène, §II. Luts et bouchons inséré dans l'article IV « De la conservation des prépats anatomiques", Traité pratique et théorique d'anatomie comparative : comprenant l'art de disséquer les animaux de toutes les classes et les moyens de conserver les pièces anatomiques, I, Paris, Méquignon-Marvis fils, 1842, p.170-171.

75. « On the Modes of Preserving the bodies of animals in Fluids of different Kinds, particularly during Voyages and Travels », The Edinburgh New Philosophical Journal, n¹0, 1828, p.160-166.

76. BOWDICH Thomas Edward and BOWDICH LEE, Sarah Wallis, Taxidermy: or, the art of collecting, preparing, and mounting objects of natural history, 4e edition, 1829 ; KNOX Frederick John, The Anatomist's Instructor, and Museum Companion: Being Practical Instructions for the Formation and Subsequent Management of Anatomical Museums, Edinburgh, Adam and Charles Black, 1836, p.134-135 ; SWAINSON William, Taxidermy, bibliography and biography, 1840, p.52-53 ; "Preservation of fish", The Magazine of Science, III, 1842, p.373-374 ; BOYD DAVIES James, The Practical Naturalist's Guide: Containing Instructions for Collecting, Preparing and Preserving Specimens, Edinburgh, MacLachlan \& Stewart, 1872, p.70.

77. BROWN Thomas, The Taxidermist's Manual: Or, The Art of Collecting, Preparing, and Preserving Objects of Natural History, Glasgow, A. Fullarton, 1833 ( $1^{\text {ère }}$ edition $), 1870\left(21^{\mathrm{e}}\right.$ edition).

78. M. Browne, “N³0-'Lithocolle’ for sealing bottles”, Practical Taxidermy: A Manual of Instruction to the Amateur in Collecting, Preserving, and Setting Up Natural History Specimens of All Kinds: to which is Added a Chapter Upon the Pictorial Arrangement of Museums, Londres, L.Upcott Gill, 1884, p.83-84 ; "N '75-'M. Peron's lithocolle' for sealing bottles", Artistic and Scientific Taxidermy and Modelling, Londres, Adam and Charles Black, 1896, p.95.

79. DONKERVOLCKE Monique, BURNY, Franz, et MUSTER Dominique, "Tissues and bone adhesives-historical aspects ", Biomaterials, vol. XIX, n¹6, 1998, p.1461-1466.

80. MAISSIAT, Jacques, «Sur un moyen de fermer exactement les vases destinés aux collections d'histoire naturelle... ", Comptes rendus hebdomadaires des séances de l'Académie des sciences, XXIV, 10, Paris, Bachelier, 1847, p. 353-355.

81. On peut penser que ces bocaux faisaient partie du paquet cacheté présenté par Maissiat la semaine précédente et dont l'Académie notifia le dépôt lors de sa séance du $1^{\mathrm{er}}$ mars 1847 , Comptes rendus hebdomadaires des séances de l'Académie des sciences, XXIV, 9, Paris, Bachelier, 1847, p. 309.

82. Voir PARMENTIER, F. "Sur une manière facile de faire l'expérience du marteau d'eau », Journal de Physique Théorique et Appliquée, $3^{\text {e }}$ série, t.I, Septembre, 1892, p. 393-394.

83. BECQUEREL, Edmond, "Rapport sur un mode de fermeture hermétique des vases, et spécialement de ceux destinés à la conservation des substances alimentaires, proposé par M. 
Maissiat », Bulletin de la Société d'encouragement pour l'industrie nationale, Vol. 46, Paris, Madame Veuve Bouchard-Huzard, 1847, p.49-50.

84. HOUEL, Charles-Nicolas, Avant-propos, p.XXIX-XXXI, Manuel d'anatomie pathologique générale et appliquée contenant la description et le cataloqgue du Musée Dupuytren, Paris, Germer Baillière, 1862, p.XXX.

85. BECQUEREL Edmond, «Rapport sur un mode de fermeture hermétique des vases, et spécialement de ceux destinés à la conservation des substances alimentaires, proposé par M. Maissiat ", Bulletin du Musée de l'industrie, vol. 12, Bruxelles, Deltombe, 1847, p.279-280 ; PAYEN, Anselme, Précis de chimie industrielle à l'usage des écoles préparatoires aux professions industrielles, des fabricants et des agriculteurs, Paris, Hachette, 1851, p.685 ; MALAGUTI Faustin-J., Leçons élémentaires de chimie, deuxième partie, Paris, Dezobry et E. Magdeleine, 1853, p.260 ; DORVAULT François, "Mastics, Luts et Ciments divers", L'Officine ou Répertoire général de pharmacie pratique, Labé, Paris, 1858, p.876. ; PAYEN Anselme et VINCENT Camille, Précis de chimie industrielle, t.I, Paris, Hachette, 1877, p.230.

86. VAILLANT Léon, «La Ménagerie des reptiles au 31 décembre 1909 », Bulletin du Muséum national d'Histoire naturelle, vol.16, $\mathrm{n}^{\circ} 1,1910$, p.11-14.

87. Poudre noire composée d'ivoire calciné et pulvérisé finement.

88. DASZKIEWICZ Piotr, «La maison Verreaux au XIX ${ }^{\mathrm{e}}$ siècle à Paris, plaque tournante des collections naturalistes mondiales ", Journal d'agriculture traditionnelle et de botanique appliquée, 39, 2, 1997, p. 111-129; GOURAUD Christophe, CHEVRIER Laurent, MEARNS Richard, « Charles and Emile Parzudaki : Natural History Dealers in Nineteenth-Century Paris ", Archives of natural history , vol.43, n¹, 2016, p. 76-89.

89. VAILLANT Léon, "De l'Étiquetage des reptiles et des poissons", extrait du numéro $1^{\mathrm{er}}$ novembre 1893, Le Naturaliste, Paris, Bureaux du journal, 1893.

90. DEYROLLE, Instruments pour les sciences naturelles, Paris, Les Fils d'Emile Deyrolle, 1931.

91. Voir entre autres, PEQUIGNOT Amandine, «Les spécimens naturalisés du Cabinet du Roy : les vestiges d'une taxidermie au XVIIIe siècle ", La Revue du Musée des Arts et Métiers, n³3, 2001, p.42-48; MARTE Fernando, PEQUIGNOT Amandine et VON ENDT David, « Arsenic in Taxidermy collections: History, Detection and Management ", Collection Forum, vol.21, n²1-2, 2006, p.143-150 ; PEQUIGNOT Amandine, "Les traitements pesticides dans les herbiers, un danger potentiel à prendre en compte ", Support Tracé, $\mathrm{n}^{\circ} 8,2008, \mathrm{p} .62-69$.

92. Voir ALBERTI Samuel, « Object and the Museum, Focus: Museums and the history of Science ", ISIS, n96, 2005, p.559-571; ALBERTI Samuel, « Construction nature behind the glass ", Museum and Society, vol.6, $\mathrm{n}^{\circ} 2,2008$, p.73-97 ; DEGUEURCE Christophe, Honoré Fragonard et ses écorchés. Un anatomiste au Siècle des lumières, Paris, Réunion des Musées Nationaux, 2010 ; LOURENCO Marta, GESSNER Samuel, « Documenting collections: cornerstones for more history of science museums ", Science and Education, vol.23, n ${ }^{\circ}$, 2014, p.727-745; PINSON Claire, Corps à corps. Les modèles anatomiques entre art et science, Paris, Mare \& Martin, 2009.

93. DAUBENTON, Louis-Jean-Marie, L'Histoire Naturelle générale et particulière: avec la Description du Cabinet du Roy, III, Paris, Imprimerie Royale, 1749, p.177.

94. PEQUIGNOT Amandine "The History of Taxidermy, "clues for the conservation" ", Collections: A Journal for Museum and Archives Professionals, 2, 3, 2006, p.245-255 ; PEQUIGNOT Amandine, « The rhinoceros of King Louis XV (1770-1793) and its horns ", Archives of natural history, vol.40, $\mathrm{n}^{\circ} 2$, 2013, p.213-227. 


\section{RÉSUMÉS}

Dans la course effrénée à la collecte naturaliste, les collections d'histoire naturelle conservée en fluide se développent prodigieusement au XVIIIe siècle en raison d'une préparation simple qui se résumerait à plonger le spécimen dans un fluide conservateur et de fermer le flacon. Or, cette évidente facilité est relativisée face à un des problèmes majeurs identifié à l'époque: l'évaporation du fluide. Le lutage, technique de ciment pour sceller hermétiquement le vase, va être un enjeu dans la conservation des collections et le témoin d'une variabilité technique entre des pratiques développées par des naturalistes de cabinet où celui-ci participe au décorum, et les expériences des voyageurs-naturalistes où il doit résister à toutes les conditions de terrain. Par ailleurs, sa transformation matérielle à travers les années esquisse une première étude chronologique et typologique qui interroge l'axiologie de l'objet dans la collection scientifique.

In the frenzied race for naturalistic collecting, natural history collections preserved in fluid was prodigiously developed in the eighteenth century thankfully to a simple preparation that would be summed up to immerse the specimen in a preservative fluid and close the bottle. However, this obvious facility has to be relativized in the face of one of the major problems identified at that time: the evaporation of the fluid. The sealing, cement technique to hermetically seal a vase, is an issue in the conservation of the collections and the witness of a technical variability between practices developed by naturalists in cabinet where the latter participates in the decorum and the experiences of the naturalist-travelers where it must withstand all field conditions. Besides, its material transformation throughout the years sketches a first chronological and typological study which questions the axiology of the objet in scientific collection

\section{INDEX}

Mots-clés : histoire des techniques, collections, histoire naturelle, musée, France, XVIIIe siècle Index chronologique : Époque moderne Index géographique : France

Keywords : history of technology, naturalists collection, museum, France, 18th century Thèmes : Un objet une technique

\section{AUTEUR}

\section{AMANDINE PEQUIGNOT}

Amandine Péquignot est maître de conférences du Muséum national d'Histoire naturelle de Paris en muséologie des sciences (UMR208 PALOC, Département Hommes et Environnement). Depuis une vingtaine d'années, elle développe ses thèmes de recherche sur l'histoire des techniques et des collections naturalistes comme les spécimens naturalisés et la taxidermie, les traitements employés pour les herbiers, les organismes préservés en fluide, en France et à l'étranger, et 
sollicite cette approche historique pour une meilleur compréhension de ce patrimoine en termes de conservation-restauration. 